\title{
Ectoderm-Derived Frontal Bone Mesenchymal Stem Cells Promote Traumatic Brain Injury Recovery by Alleviating Neuroinflammation and Glutamate Excitotoxicity
}

Qiaozhen Qin

Beijing University of Technology

Ting Wang

Institute of Basic Medical Sciences

Zhenhua Xu

Institute of Basic Medical Sciences

Shuirong Liu

Institute of Basic Medical Sciences

Heyang Zhang

Institute of Basic Medical Sciences

Zhangzhen Du

Institute of Basic Medical Sciences

Jianing Wang

Institute of Basic Medical Sciences

Yadi Wang

Institute of Basic Medical Sciences

Zhenning Wang

Institute of Basic Medical Sciences

Shanshan Yuan

Institute of Basic Medical Sciences

Jiamei Wu

Institute of Basic Medical Sciences

Wenyan $\mathrm{He}$

Institute of Basic Medical Sciences

changzhen Wang

Institute of Basic Medical Sciences

Xinlong Yan

Beijing University of Technology

Yan Wang

Institute of Basic Medical Sciences 


\section{Xiaoxia Jiang ( $\sim$ smilovjiang@163.com )}

Beijing Institute of Basic Medical Sciences https://orcid.org/0000-0003-1401-9653

\section{Research}

Keywords: frontal bone mesenchymal stem cells, traumatic brain injury, neuroinflammation, glutamate excitotoxicity

Posted Date: October 12th, 2021

DOl: https://doi.org/10.21203/rs.3.rs-951442/v1

License: (c) (i) This work is licensed under a Creative Commons Attribution 4.0 International License. Read Full License 


\section{Abstract}

Traumatic brain injury (TBI) leads to cell and tissue impairment, as well as functional deficits. Stem cells promote structural and functional recovery and thus are considered as a promising therapy for various nerve injuries. Here, we aimed to investigate the role of ectoderm-derived frontal bone mesenchymal stem cells (FbMSCs) in promoting cerebral repair and functional recovery in a murine TBI model. FbMSCs showed fibroblast like morphology and osteogenic differentiation capacity. FbMSCs were CD105, CD29 positive and CD45, CD31 negative. Different from mesoderm-derived MSCs, FbMSCs highly expressed ectoderm-specific transcription factor Tfap2 $\beta$ and growth factor FGF1. FbMSC application significantly ameliorated the behavioral deficits of TBI mice and promoted neural regeneration. Immunofluorescence staining and qRT-PCR data revealed that microglial activation and astrocyte polarization to the A1 phenotype were suppressed by FbMSC application. In addition, FGF1 secreted from FbMSCs enhanced glutamate transportation by astrocytes and alleviated the cytotoxic effect of excessive glutamate on neurons. Therefore, MSCs with characteristics of FbMSCs might be good candidates for TBI therapy.

\section{Introduction}

Traumatic brain injury (TBI) affects millions of individuals worldwide ${ }^{1}$. Although the death rate of TBI has decreased, a growing living population suffers from severe motor, neural psychological, and cognitive disabilities directly related to TBI pathogenesis ${ }^{2}$. External trauma might cause structural damage, oxidative stress ${ }^{3}$, excessive excitatory neurotransmitters ${ }^{4}$, and an inflammatory cascade responsible for further cell death ${ }^{5}$, leading to anatomical and functional brain damage. Despite extensive research, there is still no ideal way for TBI therapy owing to multiple complications during pathogenesis.

Ectoderm-derived NSCs can differentiate into neural cells, astrocytes, and oligodendrocytes. Exogenous NSCs promote the proliferation and differentiation of endogenous NSCs or neural progenitor cells (NPCs) and also differentiate into functional neurons, replacing lost neurons ${ }^{6}$. Additionally, grafted NSCs promote the switch of microglial cells into an anti-inflammatory phenotype, which might regulate the microenvironment, accelerate neuroprotection, enhance hippocampal neurogenesis, improve anatomical and functional outcomes ${ }^{7}$. Despite the extensive studies on NSCs, it remains unclear whether they are an ideal candidate for clinical TBI therapy owing to their low yield and the risk of immune rejection.

MSCs are mostly isolated and cultured from bone marrow ${ }^{8}$, umbilical $\operatorname{cord}^{9}$, and adipose tissues ${ }^{10}$, which are mesoderm-derived ${ }^{11}$. In addition to their multiple differentiate into multiple tissues such as bone, cartilage, adipose, and neuronal cells, they are immune-privileged and immunomodulatory ${ }^{12}$. MSC transplantation for TBI therapy has been investigated in animal models and clinical trials, showing significant improvements in neurological function ${ }^{13}$. Previous studies have revealed that neurorestoration and not neuro-replacement, contributes to MSC transplantation-induced TBI recovery ${ }^{8}$. MSCs

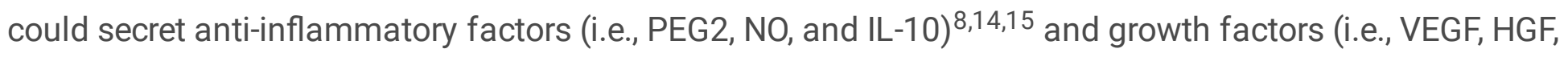
and BDNF) ${ }^{16-18}$ that suppress the inflammatory cascade, enhance neurogenesis, and promote 
synaptogenesis. Previous studies have shown that bone heals through the selective recruitment of cells from their own embryonic origins ${ }^{19,20}$. Moreover, cells derived from the same lineage are more effective in grafting procedures and promoting injury recovery ${ }^{21}$. MSCs can also be isolated from cranial, dental pulp, and frontal bone tissues, which are ectoderm-derived ${ }^{22}$. Whether these ectodermal lineage-derived MSCs might be good candidates for brain injury therapy requires further investigation.

In this study, we aimed to investigate the role and underlying mechanism of ectoderm-derived frontal bone MSCs (FbMSCs) in suppressing neuroinflammation, promoting neurogenesis, and improving cognition and behavioral deficits in TBI mice. Our study might help identify cells with FbMSCs characteristics, which could be potential candidates for TBI therapy.

\section{Materials And Methods}

\section{Animals}

Newborn or 8-10 weeks old C57BL/6N mice were purchased from Beijing Vital River Laboratory Animal Technology (Beijing). All mice were housed in a standard animal facility under controlled temperature $\left(21^{\circ} \mathrm{C}\right)$ and photoperiod $(12 \mathrm{~h}$ light $/ 12 \mathrm{~h}$ dark) with free access to water and food. All animal experiments were carried out in accordance with the "guidelines for the care and use of experimental animals" approved by Beijing Institute of Basic Medical Sciences. The ethical review committee of animal experimental institutions approved all experimental protocols.

\section{Isolation and culture of mesenchymal stem cells}

Primary bone mesenchymal stem cells (BMSCs) were isolated from murine compact bone and cultureexpanded as described in our previous report ${ }^{41}$.

Frontal bone mesenchymal stem cells (FbMSCs) and parietal bone mesenchymal stem cells (PbMSCs) were prepared from frontal or parietal bone of newborn $\mathrm{C} 57 \mathrm{BL} / 6 \mathrm{~N}$ respectively. All the meninges were thoroughly removed from bone. Bones were carefully excised into chips and transferred in a-MEM containing $10 \%$ fetal bovine serum (FBS) with the presence of $2.5 \%$ trypsin (Biological Industries; 2038152). The enzyme treated bone chips were washed three times and then cultured in a-MEM supplemented with $10 \%$ FBS and $1 \%$ penicillin/streptomycin in a humidified incubator at $37^{\circ} \mathrm{C}$ and $5 \%$ CO2. Culture medium was replaced every 2 days. Passage was twice per week at a split ratio of 1:4 or 1:3. Cells at passages 3-4 were used for the subsequent experiments.

\section{Alkaline phosphatase (ALP) and alizarin red staining}

ALP staining was performed using a NBT/BCIP staining kit (CoWin Biotech, Beijing, China) as previously described ${ }^{42}$. After osteogenic induction for 7 days, cells were fixed in $4 \%$ paraformaldehyde for 10 min, and then ALP staining was performed following the manufacturer's instructions. 
For alizarin red staining ${ }^{43}$, the cells were fixed in $95 \%$ ethanol for 30 min at room temperature after osteogenic incubation for 14 days. Subsequently, the cells were washed with distilled water and then stained with $0.1 \%$ ARS ( $\mathrm{pH}=4.2$; Sigma-Aldrich) for $20 \mathrm{~min}$.

\section{Flow cytometry analysis}

Antibodies against CD29, CD105, CD31, and CD45 were purchased from Biolegend. Data were collected on a FACS Canto II (BD) and were analyzed with FlowJo software (TreeStar).

\section{Traumatic brain injury (TBI) surgery}

Animals were subjected to either TBI using a controlled cortical impact (CCI) injury model or sham control (craniotomy with no injury). Before $\mathrm{CCl}$ injury, each animal was intraperitoneally injected with 2,2,2Tribromoethanol (350 mg/kg, Sigma-Aldrich, T48402). Animals were placed on a stereotaxic frame attached to a temperature-controlled heating pad $\left(37^{\circ} \mathrm{C}\right)$ with their scalp depilated. After exposing the skull, a 4-mm craniectomy was performed over the cortex (3.0 mm AP and $2.0 \mathrm{~mm} \mathrm{ML}$ to bregma). A pneumatically operated metal impactor (diameter $=3 \mathrm{~mm}$ ) impacted the brain at a velocity of $3.5 \mathrm{~m} / \mathrm{s}$, reaching a depth of $1.0 \mathrm{~mm}$ below the dura mater layer, and remained in the brain for $400 \mathrm{msec}$. In Matrigel-treated TBI group and FbMSC-treated TBI group, $5 \mu$ l Matrigel (Corning, 356234) or $5 \times 10^{5}$ FbMSCs mixed with $5 \mu$ l Matrigel for each mouse was applied to the injury site respectively.

\section{Cell co-culture}

Primary cortical neurons were prepared from embryonic day 15-17 mice embryos and plated on 24 well plate pretreated with $0.1 \mathrm{mg} / \mathrm{mL}$ poly-d-lysine (Sigma, 25988-63-0) at a density of $8 \times 10^{4} / \mathrm{cm} 2$. Neurons were plated and maintained in Neurobasal medium (Gibco, A2477501) supplemented with $2 \%$ B27 and $1 \%$ GlutaMAX (Gibco, 35050079) in a humidified incubator at $37^{\circ} \mathrm{C}$ with $5 \% \mathrm{CO} 2$. Neurons cell line HT22 (CL-0595) and astrocyte cell line C8-D1A (CL-0506) were purchased from KeyGEN BioTECH.

For the co-culture experiment ${ }^{44}$, one coverslip of FbMSCs $\left(5 \times 10^{4}\right.$ cells), one coverslip of C8-D1A $\left(5 \times 10^{4}\right.$ cells) and one coverslip of neurons ( $5 \times 10^{4}$ cells) or HT22 $\left(5 \times 10^{4}\right.$ cells) were placed in one $30 \mathrm{~mm}$ dish and incubated in the maintenance medium (neural basal medium supplemented with B27 and GlutaMAX). To neutralize the function of FGF1, FGF1 neutralizing antibody (Biotechno, AF-4686) was used in the experiment. Neurons or HT22 cells were collected after two days coculture and immunofluorescence staining was carried out.

\section{Beam walk test}

To examine TBI-associated complex motor movements and coordination, a beam walking test was performed as previously described, with modifications ${ }^{45,46}$. The test was performed at 1-7 days post-TBI. Briefly, the beam was a wooden bar (length: $1200 \mathrm{~mm}$ and width: $21 \mathrm{~mm}$ ) and was placed above the ground. On another end, a black box was placed for animal acclimatization. The mice were allowed to go 
on the beam to the box and visit it for $60 \mathrm{~s}$. Thereafter, the mice were placed on the beam at a starting distance of $35 \mathrm{~cm}$ from the box. The mice were allowed to go to the box and stay there for $60 \mathrm{~s}$. The step was repeated. On the next day, the mice were placed in the box for $60 \mathrm{~s}$ and then allowed to go to the box, with the starting point initially at $35 \mathrm{~cm}$, and this was then gradually increased in terms of the distance from the box up to $100 \mathrm{~cm}$. The experiment was repeated three times to cross the beam, with the mice allowed to rest in the box for $1 \mathrm{~min}$. The mean score was calculated from the three runs for each day.

\section{Morris water maze test}

The Morris water maze (MWM) test was performed as previously described, with modifications ${ }^{47}$. The apparatus used for the MWM test consists of a circular tank filled with water. The water was made opaque by adding a non-toxic white ink. A hidden platform (10cm in diameter) was placed $1 \mathrm{~cm}$ below the water surface during the training in one quadrant of the tank. The mice were trained to memorize the position of the platform; if the animals failed to find the platform, they were guided to the platform and placed on the hidden platform for $30 \mathrm{~s}$. The training session continued for five days and the latency time was calculated. On the 6th day, the platform was removed, and a probe test was conducted. In the probe trial, the number of crossings, latency to the platform, and time spent in the target quadrant were considered. The data were recorded using a video tracking system (SMART, Panlab Harvard Apparatus, Bioscience Company, Holliston, MA, USA).

\section{Open field test}

In this task, anxiety is reflected by the amount of time rodents spend at the edges of the box, avoiding the center of the open field ${ }^{48}$. Total distance travelled, entries within each zone, and the time spent and the distance traveled within each zone was recorded for $20 \mathrm{~min}$. To test for exploration of a novel chamber, data was analyzed in 5 min bins.

\section{Novel object recognition test}

Fourteen days post-TBI, we used an established protocol to assess each mouse with the NOR test ${ }^{49,50}$. The experiments were carried out 14 days post-TBI, and the mouse was placed in the box and habituated for $10 \mathrm{~min}$. On the next day, two identical novel objects (yellow cubes, cuboid) were placed in the arena, and the mouse was allowed to explore the area for $10 \mathrm{~min}$. After $1 \mathrm{~h}$, novel object (blue cubes, cylinder) and yellow cube were placed in the box, and the mouse was allowed to explore for 5 min while being recorded by camera. We recorded the total touch of exploring the novel objects.

\section{Quantitative RT-PCR}

Cells were collected in Trizol(Invitrogen, 10296010) and total RNA was prepared by chloroform extraction and isopropanol precipitation according to the manufacturer's recommendations ${ }^{51}$. Total RNA converted to cDNAs using an 5×RT Master Mix (Toyobo, 037400). Quantitative PCR was performed with $2 \times 15$ Fast qPCR Mix (SYBR)(TSINGKE, TSE202). Each amplification cycle consisted of an initial step at $95^{\circ} \mathrm{C}(5$ 
min), followed by 40 cycles of denaturation at $95^{\circ} \mathrm{C}$ for $15 \mathrm{~s}$ and annealing at $60^{\circ} \mathrm{C} 1 \mathrm{~min}$, and extension at $72{ }^{\circ} \mathrm{C}$ for $30 \mathrm{~s}$. All samples were amplified in duplicate, and every experiment was repeated at least independently 3 times. Relative gene expression was converted using the $2-\Delta \triangle \mathrm{Ct}$ method against the internal control, GAPDH.

\section{Immunofluorescence}

The immunocytochemistry is performed as described previously. Brain sections were first pretreated in $0.5 \%$ Triton X-100 in PBS for 1 hour, followed by incubation in $10 \%$ normal donkey serum and $0.1 \%$ Triton $\mathrm{X}-100$ in PBS for 1 hour. Primary antibodies were incubated with brain slices overnight at $4^{\circ} \mathrm{C}$. After additional washing in PBS, the samples were incubated with appropriate secondary antibodies conjugated to Alexa Fluor 488, Alexa Fluor cy3 for 1 hour at room temperature, and then incubated with DAPI (Thermo Fisher, D1306) for 10 min, followed by washing in PBS.

\section{Cell viability staining}

We used the live-dead cell imaging kit (Thermo Fisher, L3224) to evaluate cell viability ${ }^{52}$.

\section{Glutamate assay}

Glutamate assay kit (Invitrogen, MAK004-1KT) from Sigma was used to quantify glutamate concentration in $\mathrm{C} 8$ cells according to the manufacturer's instruction ${ }^{53,54}$.

\section{Statistical analysis}

Data are presented as mean \pm SEM. Two-way ANOVA was used to analyze all behavioral tests between and among the treatment groups. In anatomical and biochemical studies, one-way or two-way ANOVA was used to compare multiple groups. A Bonferroni post hoc analysis was used to determine whether differences were significant. Differences between two groups were tested with the two-tailed Student's ttest. The criteria for statistical significance were $p<0.05$.

\section{Results}

\section{Characteristics of ectoderm-derived FbMSCs}

Ectoderm-derived FbMSCs isolated from the frontal bone of neonatal mice exhibited fibroblast-like morphology (Fig. 1A, B). After cultured in osteoblast induction conditions, FbMSCs were positive for ALP and ARS staining (Fig. 1C). Flow cytometric analysis showed that FbMSCs were positive for mesenchymal makers CD29 and CD105, but negative for hematopoietic and endothelial cell lineage markers CD45 and CD31, respectively (Fig. 1D). To investigate the potential immunoregulatory function, FbMSCs were stimulated with different factors. As shown in Fig. 1E, the expression of iNOS, IL-10, IL-6, and HGF was significantly increased with TNFa and IFNy stimulation. Transcriptomic analysis using an Affymetrix Clariom D array was performed to examine the transcripts in FbMSCs and bone marrow MSCs 
(BMSCs). Heat map assays identified the differences of gene expression between FbMSCs and BMSCs. The ectodermal-specific transcription factor Tfap2 $\beta$ and growth factor FGF1 were highly expressed in FbMSCs (Fig. 1F), which was confirmed by qRT-PCR analysis (Fig. 1I). GO and KEGG enrichment analysis demonstrated differences in nervous system-related pathways such as neuroactive ligand-receptor interaction and axon guidance (Fig. 1G, H), which were confirmed by qRT-PCR analysis (Fig. $1 \mathrm{~J}, \mathrm{~K}$ ). Microarray analysis was also performed on FbMSCs and parietal bone derived MSCs (PbMSCs), which were mesoderm-derived. Compared to PbMSCs, FbMSCs also showed a higher expression of Tfap2 $\beta$ and FGF1 (sFig. 1).

\section{FbMSC application improves learning and memory deficits in TBI mice}

We explored the therapeutic effects of FbMSCs in the cortical impact injury (CCI) TBI mouse model. $\mathrm{CCl}$ was performed on adult C57BL/6N mice in a moderate TBI model. After brain injury, mice showed deficient learning and memory abilities (sFig. 2), increased Iba1 and GFAP immunofluorescence staining intensity, and decreased microtubule associated protein 2 (MAP2) staining (sFig. 3). FbMSCs mixed with Matrigel or Matrigel alone were transplanted to the injury site immediately after the injury. Various behavioral experiments, including beam walk test, Morris water maze (MWM) test, open field test, and new object recognition experiment, were conducted to examine the spatial learning and memory abilities of all the four TBI groups (Sham group, TBI alone, Matrigel-treated TBI, and FbMSC-treated TBI; Fig. 2A). The movement and coordination abilities of all groups were evaluated using the beam walk test, which revealed that the FbMSC-treated TBI group had an improved motor coordination ability (Fig. 2B). Typical escape routes in the MWM test were shown in Fig. 2C. The escape latency of the FbMSC-treated TBI group was similar to that of the Sham group but much faster than that of the TBI group and Matrigeltreated TBI group (Fig. 2D, E). However, no significant differences were identified in movement speed among the four groups (Fig. 2F). In the open field test, the movement speed and the total distances in the field were similar among the four groups (Fig. 2G, H). The novel object recognition test is a learning and memory test based on the principle that animals have a natural tendency to explore novel object ${ }^{23}$. The ability of the FbMSC-treated TBI group to explore new objects was significantly improved, compared with that of the TBI group (Fig. 2I). These results suggested that FbMSC transplantation could improve the learning and cognitive function of TBI mice, as well as their motor coordination ability to a certain extent.

\section{Transcriptional profile after FbMSC transplantation}

Corresponding to the improved behavior, the wound size and area were both significantly reduced to healthy levels with FbMSC transplantation, whereas the TBI group only showed partial recovery (Fig. 3A\&B). To further clarify the effect of FbMSC application, blood and biochemical indices were compared among the groups and found to have no significant differences (sFig. 4). Brain tissues around the injured area from FbMSC-treated group and TBI group were collected and processed for subsequent downstream bulk RNA sequencing (RNA-seq). Overall, many differentially expressed genes were found when the transcriptomes of the both groups were compared. Volcano plot (Fig. 3C) depicted the significantly upregulated and downregulated mRNAs and some significantly up-regulated genes and down-regulated 
genes were marked. The Heatmap revealed the differences in the relative gene expression between FbMSC-treated TBI and TBI group (Fig. 3D). GO and KEGG enrichment analysis revealed significant differences in neuroactive ligand-receptor interaction and IL-17 signaling pathway between FbMSCtreated TBI and TBI group (Fig. 3E,F). Genes related to the relative pathways were confirmed by qRT-PCR analysis (Fig. 3G-I).

\section{FbMSC transplantation enhances neurogenesis and synaptic remodeling}

Neuron death and diffuse axonal injury are common complications of $\mathrm{TBI}^{24}$. To quantify mature neurons, MAP2 staining was performed at $4 \mathrm{~d}$ and $28 \mathrm{~d}$ post-injury in the injured area and hippocampus. The labeling intensity of MAP2 in the injured area and hippocampus was significantly decreased in the TBI group at $4 \mathrm{~d}$ (Fig. 4A-D) and $28 \mathrm{~d}$ (Fig. 4E-H) post-injury. The TBI group had significantly lower MAP2 staining intensities than the Sham group in both the injured area and hippocampus. MAP2 staining quantification of the FbMSC-treated group were comparable to those in the Sham group, which indicated that FbMSC transplantation dramatically promoted neuron recovery. 3D construction of MAP2 staining confirmed the recovery of neurons with FbMSC application (Fig. 4l). Newly generated neurons from hippocampal neurogenesis are thought to play a crucial role in some types of learning and memory ${ }^{25,26}$.

We used DCX to label the developing and migrating neurons. The labeling intensity of DCX in the FbMSCtreated TBI group was more robust than that in the TBI group (Fig. 4J\&K). In addition, the level of BDNF at 14 and $28 \mathrm{~d}$ post-injury was significantly higher in the FbMSC-treated TBI group than in the TBI group (Fig. 4L). These results revealed that FbMSC treatment could promote neurogenesis and synaptic remodeling.

\section{FbMSC transplantation reduces the activation of microglia}

Microglia are resident immune cells in the brain that play an important role in regulating neuroinflammation or injury. Iba1 labeling was used to evaluate microglia activation in the injured brain (Fig. 5A). Activated microglia were lba1-positive and showed enlarged cell bodies and axon coarseness. The labeling intensity of Iba 1 in the injured area and hippocampus was significantly increased in the TBI group at $4 \mathrm{~d}$ (Fig. 5B-E) and $28 \mathrm{~d}$ (Fig. 5F-I) post-injury. Immunofluorescence staining and the intensity quantification data showed that FbMSC transplantation dramatically reduced microglial activation. Aif-1 and Cx3cr1 expression levels in the injured tissues at $4 \mathrm{~d}, 14 \mathrm{~d}$ and $28 \mathrm{~d}$ after TBI were quantified to further confirm microglial activation. As shown in Fig. 5J, the expression levels of Aif-1 and Cx3cr1 were increased in the TBI group but significantly decreased in the FbMSC-treated TBI group, compared to those in the Sham group. We also examined the expression of pro-inflammatory factors in brain tissue postinjury. Overall, the levels of TNFa, IL1 $\beta$, and IL- 6 in the FbMSC-treated TBI group were significantly decreased compared to those in the TBI group (Fig. 5K). These results indicated that FbMSCs could restrict the activation of microglia to a certain extent.

\section{FbMSC application alleviates the activation of astrocytes}


Brain injury is generally accompanied by reactive astrogliosis ${ }^{27,28}$. We used glial fibrillary acidic protein (GFAP) to label astrocytes in the brain tissues of the four groups (Fig. 6A). As revealed by GFAP immunofluorescence, the appearance of astrocytes with increased sizes and enlarged branches was mostly observed in $4 \mathrm{~d}$ (Fig. 6B, C) and $28 \mathrm{~d}$ (Fig. 6F, G) in TBI and Matrigel-treated TBI groups. GFAP labeling intensity in the FbMSC-treated TBI group was almost similar to that in the Sham group but significantly lower than that in the TBI and Matrigel-treated TBI groups (Fig. 6D, E, H, I). Brain tissues around the injury site were collected to examine the activation of astrocytes using qRT-PCR. The levels of A1 astrocyte-specific transcripts H2-T23, H2-D1, and GBP2 were significantly decreased in the FbMSCtreated TBI group (Fig. $6 \mathrm{~J}$ ). Different doses of FbMSCs were co-cultured with the astrocyte cell line C8D1A and stimulated with TNFa $(150 \mathrm{ng} / \mathrm{ml})$ and IL1 $\beta(50 \mathrm{ng} / \mathrm{ml})(\mathrm{Fig} .6 \mathrm{~K})$. The results revealed that FbMSCs could significantly suppress the expression of proinflammatory factors IL-6, IL $1 \beta$, and TNFa in C8-D1A cells (Fig. 6L).

\section{FGF1 secreted from FbMSCs alleviates glutamate excitotoxicity to neurons by promoting glutamate transportation in astrocytes}

In addition to increased neuroinflammation, the excessive release of glutamate is also responsible for the secondary neuronal death in $\mathrm{TBI}^{29}$. To verify the effect of glutamate on neurons, primary neurons were treated with different doses of glutamate and then stained with MAP2. The number of cytoskeleton branches of the neurons was significantly decreased with glutamate treatment in a dose-dependent manner, with more glutamate resulting in fewer branches (Fig. 7A-C). We then treated the neuron cell line HT22 with glutamate and performed live and dead assay via calcein-AM (green, live)/ethidium homodimer (red, dead) staining. As shown in Fig. 7D\&E, more red staining appeared in cells treated with glutamate, indicating the cytotoxicity of glutamate on HT22 cells. Previous studies ${ }^{30}$ and our data demonstrated that FGF1 exposure induced the expression of GLT-1 in astrocytes (Fig 7F, H) and promoted glutamate transport by astrocytes (Fig. 7G, I). Compared with MSCs from the bone marrow and parietal bone, FbMSCs highly expressed FGF1 (Fig. 1F\&l, sFig. 1). To evaluate the influence of FGF1 from FbMSCs on astrocytes, we co-cultured FbMSCs, astrocyte cell line C8-D1A, and primary neurons in the presence of glutamate for $24 \mathrm{~h}$. MAP2 staining data and neuron branches counting revealed that the presence of FbMSCs and C8-D1A co-cultured reduced the cytotoxicity of glutamate on neurons (Fig. 7J, $\mathrm{K})$. To explore whether FGF1 secreted from FbMSCs mediated the recovery of neurological damage, a neutralizing antibody against FGF1 (FGF1 $\left.{ }^{\mathrm{Ab}}\right)(5 \mu \mathrm{g} / \mathrm{ml})$ was added to the FbMSCs, C8-D1A, and neuron co-culture system. The protective effect of FbMSCs and C8-D1A was compromised with FGF1 ${ }^{\mathrm{Ab}}$ addition (Fig. 7J, K). We also co-cultured FbMSCs, astrocyte cell line C8-D1A, and HT22 cells in the presence of glutamate for $24 \mathrm{~h}$ and performed live/dead staining. Similarly, FbMSCs and C8-D1A co-culture alleviated the cytotoxicity of glutamate on $\mathrm{HT} 22$ cells. The presence of FGF1 ${ }^{\mathrm{Ab}}$ reduced the protective effect of FbMSCs and C8-D1A (Fig. 7L, M). Collectively, these data revealed an important role of FGF1 from FbMSCs in mediating the protective effect on neurons.

\section{Discussion}


In this study, we found that ectoderm-derived FbMSC application in TBI mice significantly inhibited neuroinflammation, promoted neurogenesis, improved cognition and behavioral deficits. Additionally, FGF1 from FbMSCs enhanced glutamate transportation in astrocytes, contributing to the alleviation of glutamate cytotoxicity in neurons (Fig. 8).

TBI pathogenesis includes a complex process from primary to secondary injuries, leading to anatomical and functional brain damage ${ }^{31}$. Numerous therapeutic strategies for alleviating inflammation, scavenging reactive oxygen species, and promoting tissue regeneration have been developed for TBI recovery ${ }^{32}$. Of these, stem cell-based therapy provided an ideal therapeutic option for neurological disorders based on its ability to inhibit neuroinflammation and promote tissue reconstruction ${ }^{33}$. In the present study, we found that FbMSCs isolated from the frontal bone demonstrated some characteristics of NSCs, and were ectoderm-derived and could differentiate into different types of neurons (data not shown). FbMSCs also showed properties similar to those of MSCs, including the secretion of antiinflammatory factors under TNFa and IL1 $\beta$ stimulation and differentiation into osteoblasts. Our data showed that in situ FbMSC application suppressed microglia and astrocyte activation, promoted neural regeneration, and alleviated behavioral deficits in TBI mice. Previous studies revealed that NSC grafts could ameliorate TBI-associated histological alterations as well as neurological and cognitive deficits in a rat model ${ }^{34}$. Exogenous NSCs not only initiated endogenous NSCs or NPCs proliferation, but also differentiate into functional neurons. We found that increased MAP2- and DCX-positive cells in the hippocampus of the FbMSC-treated TBI group indicated that FbMSCs might promote NSC or NPC differentiation. However, whether FbMSCs can differentiate into functional neurons in vivo and integrate into brain tissues need to be further investigated.

Mesoderm-derived MSCs have been extensively investigated in TBI models owing to their abundance, multiple differentiation abilities, immunoregulatory capacities, and easily available from various tissues (i.e., bone marrow, umbilical cord, and adipose tissue $)^{8-10}$. Previous studies have demonstrated that stem cells from the same lineage are more effective for post-injury recovery ${ }^{19,20}$. Leucht et al. reported that neural crest stem cells contributed exclusively to the regeneration of neural crest-derived skeletal elements, indicating that reparative strategies should focus on lineage origin to maximize the effectiveness of recovery treatment ${ }^{19}$. NSCs and their derived-neurons, oligodendrocytes and astrocytes originate from the ectoderm. Here, we isolated and investigated the reparative function of ectodermderived FbMSCs in TBI mice. RNA-seq and qRT-PCR data showed that FbMSCs highly expressed the ectodermal cell-specific transcription factor Tfap2 $\beta$, which is not expressed in mesoderm-derived cells. Similar to those of bone marrow MSCs, after induction by TNFa and IL $1 \beta$, FbMSCs produced iNOS and IL10 , which have been reported to play important roles in suppressing excessive immune responses 35,36 .

In the central nervous system, excessive glutamate release is responsible for the secondary neuronal death following neuronal injury. Glutamate excitotoxicity in the acute phase of TBI generally aggravates neuronal loss. Different strategies have been explored to protect neurons from glutamate excitotoxicity. Li et al. transplanted N-acetyl-aspartyl-glutamate synthetase-activated NSCs to sustain endogenous 
glutamate levels ${ }^{37}$. VEGF from NSCs contributes to stroke recovery by promoting the upregulation of GLT1 expression in astrocytes and reducing peri-ischemic extracellular levels of glutamate ${ }^{38,39}$. In the present study, we found that FGF1 secreted from FbMSCs could enhance glutamate transportation by astrocytes. RNA-seq assays revealed that FbMSCs expressed higher levels of FGF1 than bone marrow MSCs. In vitro analysis showed that FGF1 promoted GLT-1 expression in astrocytes and enhanced glutamate transportation. Previous studies have shown that FGF1 was found in preadipocytes ${ }^{40}$, astrocytes ${ }^{30}$, and neural crest-derived osteogenic progenitors ${ }^{19}$ and could minimize the number of apoptotic cells after injury. Our results revealed that addition of FGF1 antibody attenuated the protective effect of C8-D1A and FbMSCs. FbMSCs could also differentiate into neural cells in vitro, and whether in vivo neuro-replacement after brain injury by cells derived from FbMSCs requires further investigation.

In summary, this is the first report on applying ectoderm-derived FbMSCs in a TBI model to alleviate anatomical and functional injury. FbMSCs effectively ameliorated structural and functional injury, markedly reduced neuroinflammation, alleviated excessive glutamate excitotoxicity, and mitigated neuronal damage. Additionally, FGF1 from FbMSCs promoted the transportation of glutamate by astrocytes, probably mitigating the continuous neuronal loss. Overall, our findings highlight the therapeutic potential of ectoderm-derived FbMSCs for TBI recovery.

\section{Declarations}

\section{ACKNOWLEDGMENTS}

This study was supported by grants from National Natural Science Foundation of China (No. No. 31971285 to XXJ, 81771998 to XXJ); Beijing Natural Science Foundation (No. 7202149 to XXJ); National Key Research and Development Program of China (No. 2016YFE0204400 to YW).

\section{AUTHOR CONTRIBUTIONS}

XJ conceived and designed the experiments. QQ, TW, ZX, SL, HZ, ZD, JW, ZW, SY and JW performed the experiments. QQ, TW, ZX, SL, WH, CW, XY, ZW, YX, YW and XJ analyzed experimental data. WH, CW, YW and $\mathrm{XJ}$ provided regent. QQ, $\mathrm{XY}$ and $\mathrm{XJ}$ wrote the manuscript. All authors read and approved the manuscript.

\section{COMPETING INTERESTS}

The authors declare that they have no competing interest.

\section{References}

1. Guan F, et al. The TRIM protein Mitsugumin 53 enhances survival and therapeutic efficacy of stem cells in murine traumatic brain injury. Stem Cell Res Ther. 2019;10:352. doi:10.1186/s13287-0191433-4. 
2. Zhang J, Zhang F, Dong J. Coagulopathy induced by traumatic brain injury: systemic manifestation of a localized injury. Blood. 2018;131:2001-6. doi:10.1182/blood-2017-11-784108.

3. Cornelius $\mathrm{C}$, et al. Traumatic brain injury: oxidative stress and neuroprotection. Antioxid Redox Signal. 2013;19:836-53. doi:10.1089/ars.2012.4981.

4. Dorsett C, et al. Glutamate Neurotransmission in Rodent Models of Traumatic Brain Injury. J Neurotrauma. 2017;34:263-72. doi:10.1089/neu.2015.4373.

5. Erny D, et al. Host microbiota constantly control maturation and function of microglia in the CNS. Nature neuroscience. 2015;18:965-77. doi:10.1038/nn.4030.

6. Gao J, et al. Human Neural Stem Cell Transplantation-Mediated Alteration of Microglial/Macrophage Phenotypes after Traumatic Brain Injury. Cell Transplant. 2016;25:1863-77. doi:10.3727/096368916x691150.

7. Betancur M, et al. Chondroitin Sulfate Glycosaminoglycan Matrices Promote Neural Stem Cell Maintenance and Neuroprotection Post-Traumatic Brain Injury. ACS biomaterials science engineering. 2017;3:420-30. doi:10.1021/acsbiomaterials.6b00805.

8. Cho $D$, et al. Mesenchymal stem cells reciprocally regulate the M1/M2 balance in mouse bone marrow-derived macrophages. Exp Mol Med. 2014;46:e70. doi:10.1038/emm.2013.135.

9. Jia Y, et al. HGF Mediates Clinical-Grade Human Umbilical Cord-Derived Mesenchymal Stem Cells Improved Functional Recovery in a Senescence-Accelerated Mouse Model of Alzheimer's Disease. Advanced science (Weinheim, Baden-Wurttemberg, Germany) 7, 1903809, doi:10.1002/advs.201903809 (2020).

10. Wang M, Song L, Strange C, Dong X, Wang H. Therapeutic Effects of Adipose Stem Cells from Diabetic Mice for the Treatment of Type 2 Diabetes. Molecular therapy: the journal of the American Society of Gene Therapy. 2018;26:1921-30. doi:10.1016/j.ymthe.2018.06.013.

11. Gargett C, Schwab K, Deane J. Endometrial stem/progenitor cells: the first 10 years. Hum Reprod Update. 2016;22:137-63. doi:10.1093/humupd/dmv051.

12. Shi $Y$, et al. Immunoregulatory mechanisms of mesenchymal stem and stromal cells in inflammatory diseases. Nat Rev Nephrol. 2018;14:493-507. doi:10.1038/s41581-018-0023-5.

13. Huang $\mathrm{H}$, Chen Lin, Mao G, Sharma $\mathrm{H}$. Clinical neurorestorative cell therapies: Developmental process, current state, and future prospective. J Neurorestoratology. 2020;8(2):61-82.

14. Ren J, et al. Intranasal delivery of MSC-derived exosomes attenuates allergic asthma via expanding IL-10 producing lung interstitial macrophages in mice. Int Immunopharmacol. 2021;91:107288. doi:10.1016/j.intimp.2020.107288.

15. Pajarinen J, et al. Mesenchymal stem cell-macrophage crosstalk and bone healing. Biomaterials. 2019;196:80-9. doi:10.1016/j.biomaterials.2017.12.025.

16. Shi W, et al. BDNF blended chitosan scaffolds for human umbilical cord MSC transplants in traumatic brain injury therapy. Biomaterials. 2012;33:3119-26. doi:10.1016/j.biomaterials.2012.01.009. 
17. Anderson $\mathrm{M}$, et al. Required growth facilitators propel axon regeneration across complete spinal cord injury. Nature. 2018;561:396-400. doi:10.1038/s41586-018-0467-6.

18. An Y, et al. Autophagy promotes MSC-mediated vascularization in cutaneous wound healing via regulation of VEGF secretion. Cell death disease. 2018;9:58. doi:10.1038/s41419-017-0082-8.

19. Kidwai F, et al. Lineage-specific differentiation of osteogenic progenitors from pluripotent stem cells reveals the FGF1-RUNX2 association in neural crest-derived osteoprogenitors. Stem Cells. 2020;38:1107-23. doi:10.1002/stem.3206.

20. Leucht $P$, et al. Embryonic origin and Hox status determine progenitor cell fate during adult bone regeneration. Development. 2008;135:2845-54. doi:10.1242/dev.023788.

21. Armstrong R, Mierzwa A, Sullivan G, Sanchez M. Myelin and oligodendrocyte lineage cells in white matter pathology and plasticity after traumatic brain injury. Neuropharmacology. 2016;110:654-9. doi:10.1016/j.neuropharm.2015.04.029.

22. Hochuli A, Senegaglia A, Selenko A, Fracaro L, Brofman P. Dental Pulp From Human Exfoliated Deciduous Teeth-Derived Stromal Cells With Demonstrated Neuronal Potential: In Vivo And In Vitro Studies. Curr Stem Cell Res Therapy. 2021. doi:10.2174/1574888x16666210215160402.

23. Leger M, et al. Object recognition test in mice. Nature protocols. 2013;8:2531-7. doi:10.1038/nprot.2013.155.

24. Hao P, et al. Neural repair by NT3-chitosan via enhancement of endogenous neurogenesis after adult focal aspiration brain injury. Biomaterials. 2017;140:88-102. doi:10.1016/j.biomaterials.2017.04.014.

25. Hambright W, Fonseca R, Chen L, Na R, Ran Q. Ablation of ferroptosis regulator glutathione peroxidase 4 in forebrain neurons promotes cognitive impairment and neurodegeneration. Redox Biol. 2017;12:8-17. doi:10.1016/j.redox.2017.01.021.

26. Liu Y, Brent G. Thyroid hormone and the brain: Mechanisms of action in development and role in protection and promotion of recovery after brain injury. Pharmacol Ther. 2018;186:176-85. doi:10.1016/j.pharmthera.2018.01.007.

27. Anderson $\mathrm{M}$, et al. Astrocyte scar formation aids central nervous system axon regeneration. Nature. 2016;532:195-200. doi:10.1038/nature17623.

28. Karve I, Taylor J, Crack P. The contribution of astrocytes and microglia to traumatic brain injury. $\mathrm{Br} \mathrm{J}$ Pharmacol. 2016;173:692-702. doi:10.1111/bph.13125.

29. Dorsett C, et al. Traumatic Brain Injury Induces Alterations in Cortical Glutamate Uptake without a Reduction in Glutamate Transporter-1 Protein Expression. J Neurotrauma. 2017;34:220-34. doi:10.1089/neu.2015.4372.

30. Roybon $L$, et al. Human stem cell-derived spinal cord astrocytes with defined mature or reactive phenotypes. Cell reports. 2013;4:1035-48. doi:10.1016/j.celrep.2013.06.021.

31. Siebold L, Obenaus A, Goyal R. Criteria to define mild, moderate, and severe traumatic brain injury in the mouse controlled cortical impact model. Exp Neurol. 2018;310:48-57. doi:10.1016/j.expneurol.2018.07.004. 
32. Yun $S$, et al. Block of $A 1$ astrocyte conversion by microglia is neuroprotective in models of Parkinson's disease. Nature medicine. 2018;24:931-8. doi:10.1038/s41591-018-0051-5.

33. Clinical neurorestorative cell therapies. Developmental process, current state and future prospective. doi:10.26599/JNR.2020.9040009.

34. Pang A, et al. Neural Stem Cell Transplantation Is Associated with Inhibition of Apoptosis, Bcl-xL Upregulation, and Recovery of Neurological Function in a Rat Model of Traumatic Brain Injury. Cell Transplant. 2017;26:1262-75. doi:10.1177/0963689717715168.

35. Torrisi J, et al. Inhibition of Inflammation and iNOS Improves Lymphatic Function in Obesity. Scientific reports. 2016;6:19817. doi:10.1038/srep19817.

36. Wang X, Wong K, Ouyang W, Rutz S. Targeting IL-10 Family Cytokines for the Treatment of Human Diseases. Cold Spring Harb Perspect Biol 11, doi:10.1101/cshperspect.a028548 (2019).

37. Li M, et al. Transplantation of $\mathrm{N}$-acetyl aspartyl-glutamate synthetase-activated neural stem cells after experimental traumatic brain injury significantly improves neurological recovery. Cellular physiology biochemistry: international journal of experimental cellular physiology biochemistry pharmacology. 2013;32:1776-89. doi:10.1159/000356611.

38. Guo Y, et al. The effects of astrocytes on differentiation of neural stem cells are influenced by knockdown of the glutamate transporter, GLT-1. Neurochem Int. 2013;63:498-506. doi:10.1016/j.neuint.2013.08.003.

39. Bacigaluppi M, et al. Neural Stem Cell Transplantation Induces Stroke Recovery by Upregulating Glutamate Transporter GLT-1 in Astrocytes. The Journal of neuroscience: the official journal of the Society for Neuroscience. 2016;36:10529-44. doi:10.1523/jneurosci.1643-16.2016.

40. Kim Y, et al. Identification of carboxypeptidase X (CPX)-1 as a positive regulator of adipogenesis. FASEB journal: official publication of the Federation of American Societies for Experimental Biology. 2016;30:2528-40. doi:10.1096/fj.201500107R.

41. Zhu H, et al. A protocol for isolation and culture of mesenchymal stem cells from mouse compact bone. Nature protocols. 2010;5:550-60. doi:10.1038/nprot.2009.238.

42. Wang $Y$, et al. Adiponectin regulates BMSC osteogenic differentiation and osteogenesis through the Wnt/ $\beta$-catenin pathway. Scientific reports. 2017;7:3652. doi:10.1038/s41598-017-03899-z.

43. Li X, et al. Circular RNA CDR1as regulates osteoblastic differentiation of periodontal ligament stem cells via the miR-7/GDF5/SMAD and p38 MAPK signaling pathway. Stem Cell Res Ther. 2018;9:232. doi:10.1186/s13287-018-0976-0.

44. Ye X, et al. Astrocytic Lrp4 (Low-Density Lipoprotein Receptor-Related Protein 4) Contributes to Ischemia-Induced Brain Injury by Regulating ATP Release and Adenosine-AR (Adenosine A2A Receptor) Signaling. Stroke. 2018;49:165-74. doi:10.1161/strokeaha.117.018115.

45. Rehman S, et al. Neurological Enhancement Effects of Melatonin against Brain Injury-Induced Oxidative Stress, Neuroinflammation, and Neurodegeneration via AMPK/CREB Signaling. Cells 8, doi:10.3390/cells8070760 (2019). 
46. Chen M, et al. Inhibition of miR-331-3p and miR-9-5p ameliorates Alzheimer's disease by enhancing autophagy. Theranostics. 2021;11:2395-409. doi:10.7150/thno.47408.

47. Khrimian L, et al. Gpr158 mediates osteocalcin's regulation of cognition. The Journal of experimental medicine. 2017;214:2859-73. doi:10.1084/jem.20171320.

48. Yang $Y$, et al. Ketamine blocks bursting in the lateral habenula to rapidly relieve depression. Nature. 2018;554:317-22. doi:10.1038/nature25509.

49. Sawangjit $A$, et al. The hippocampus is crucial for forming non-hippocampal long-term memory during sleep. Nature. 2018;564:109-13. doi:10.1038/s41586-018-0716-8.

50. Lee A, et al. Prkcz null mice show normal learning and memory. Nature. 2013;493:416-9. doi:10.1038/nature11803.

51. Ziller $\mathrm{M}$, et al. Dissecting neural differentiation regulatory networks through epigenetic footprinting. Nature. 2015;518:355-9. doi:10.1038/nature13990.

52. Pattarone G, Acion L, Simian M, larussi E. Learning deep features for dead and living breast cancer cell classification without staining. Scientific reports. 2021;11:10304. doi:10.1038/s41598-02189895-w.

53. Shah S, et al. Anthocyanins abrogate glutamate-induced AMPK activation, oxidative stress, neuroinflammation, and neurodegeneration in postnatal rat brain. J Neuroinflamm. 2016;13:286. doi:10.1186/s12974-016-0752-y.

54. Barbar L, et al. CD49f Is a Novel Marker of Functional and Reactive Human iPSC-Derived Astrocytes. Neuron 107, 436-453.e412, doi:10.1016/j.neuron.2020.05.014 (2020).

\section{Figures}


A.

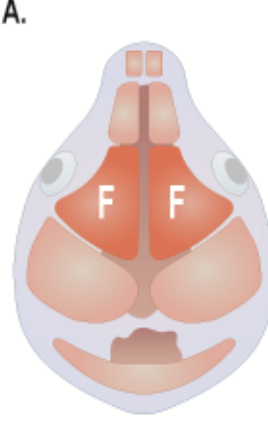

B.
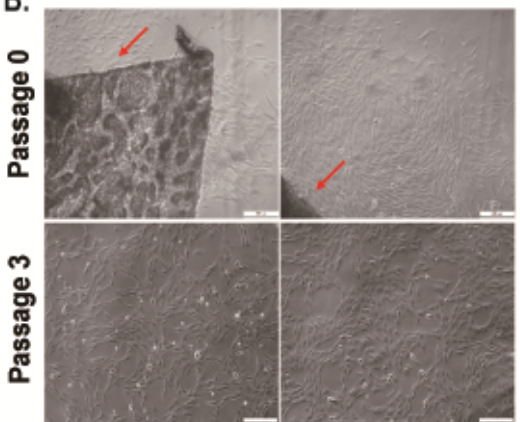

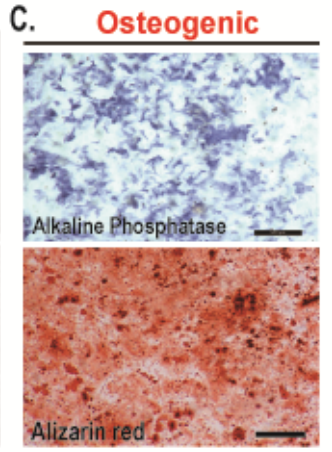

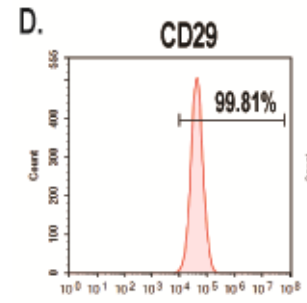

E.
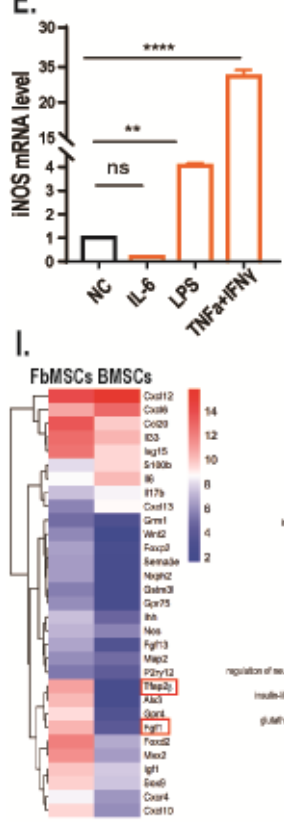

L.

M.

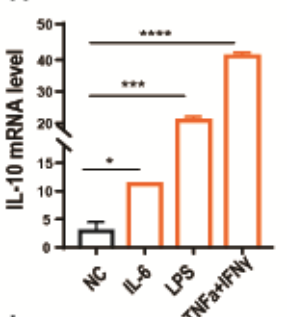

J.
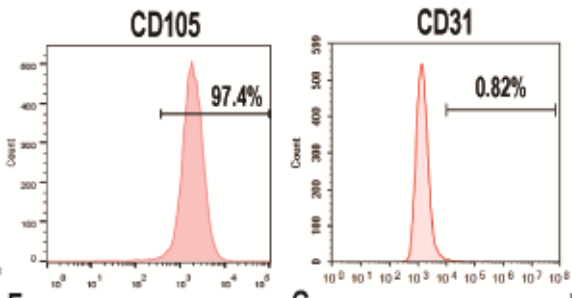

G.

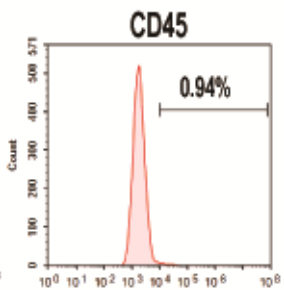

$\mathrm{H}$.
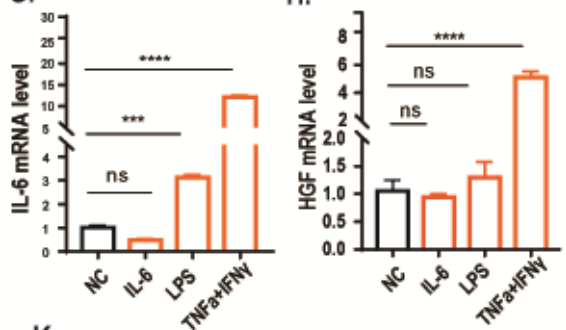

K.

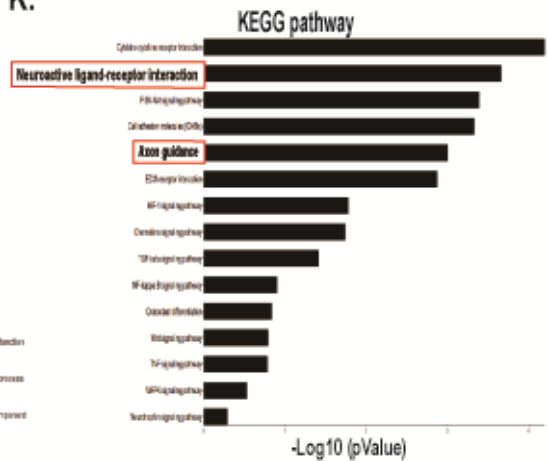

N. Axon guidance pattwway MAPK pathway
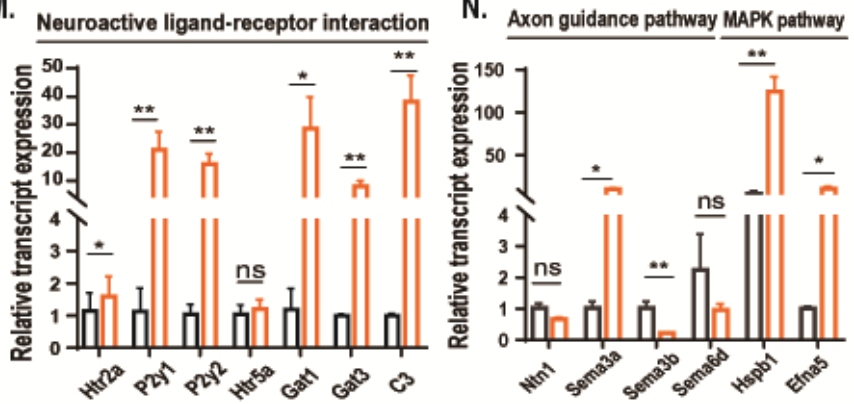

\section{Figure 1}

Characteristics of frontal bone mesenchymal stem cells (FbMSCs). (A) Schematic of murine skull. Areas marked F refer to frontal bone, from where FbMSCs were isolated. (B) Representative morphological features of FbMSCs. Scale bar, $200 \mu \mathrm{m}$. (C) ALP and ARS staining of FbMSCs. Scale bar, $500 \mu \mathrm{m}$. (D) Flow cytometric analysis of FbMSCs. Expression of iNOS (E), IL-10 (F), IL-6 (G), and HGF (H) in FbMSCs stimulated with the indicated factors. Microarray analysis was performed on FbMSCs and bone marrow 
MSCs (BMSCs). (I) Cluster heat map of representative differential genes in FbMSCs and BMSCs. GO (J) and KEGG (K) analysis of enriched pathway in FbMSCs. Quantitative RT-PCR verified the higher expression of Tfap2 $\beta, F g f 1$, and $\mathrm{Grm} 1(\mathrm{~L})$, as well as genes related to the neuron ligand-receptor pathway $(\mathrm{M})$, axon guidance pathway and MAPK pathway (N) in FbMSCs. Data are presented as the mean \pm standard error; ${ }^{*}, * *, * * *$, and $* * * *$ indicate significance at $p<0.05, p<0.01, p<0.001$, and $p<0.0001$, respectively.

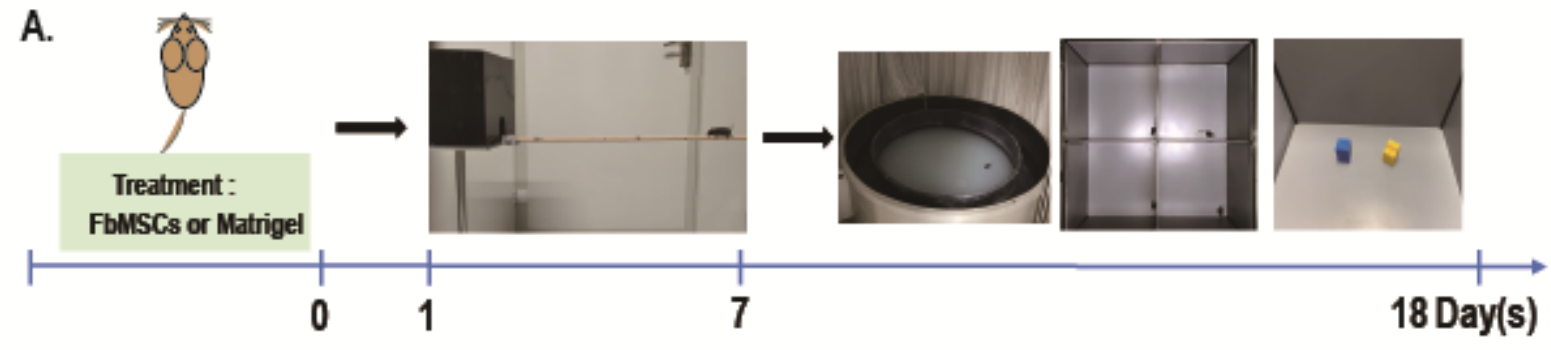

B.

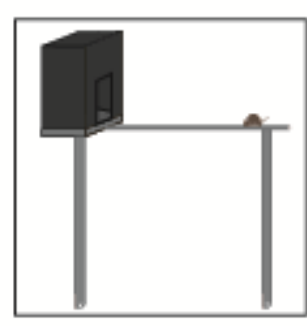

D.

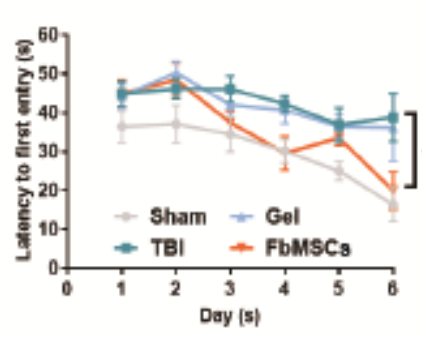

E.
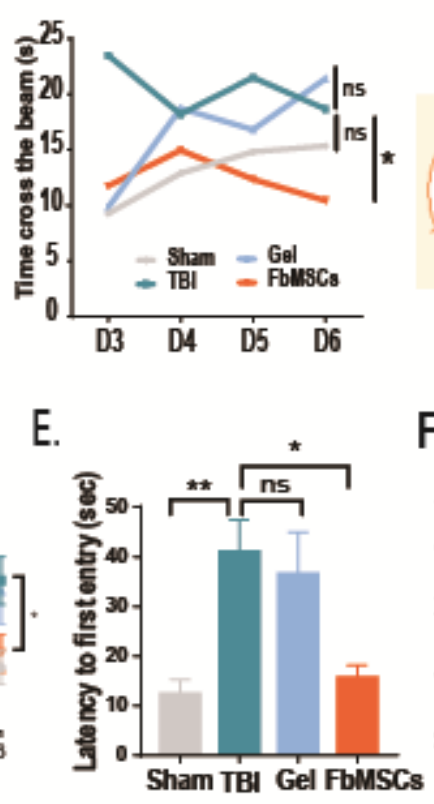

C.

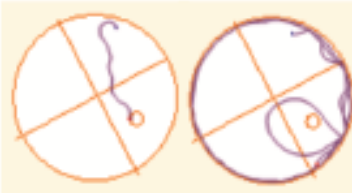

Sham

TBI
Morris Water Maze Test

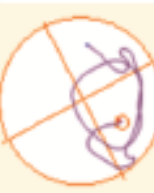

Gel

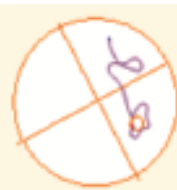

FbMSCs

H. Open field test

I.

F.

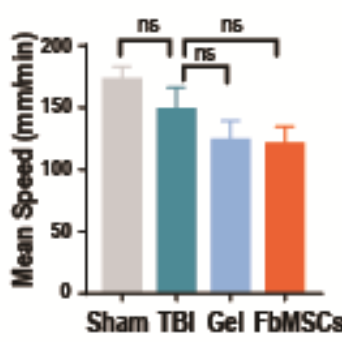

G. Open field test

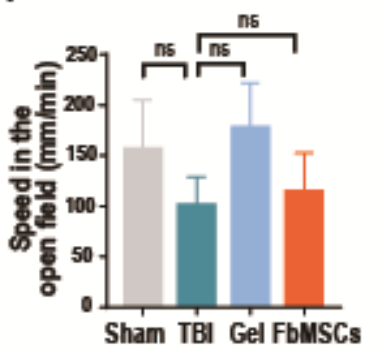

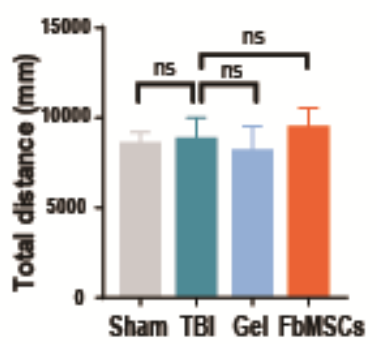

New object recognition
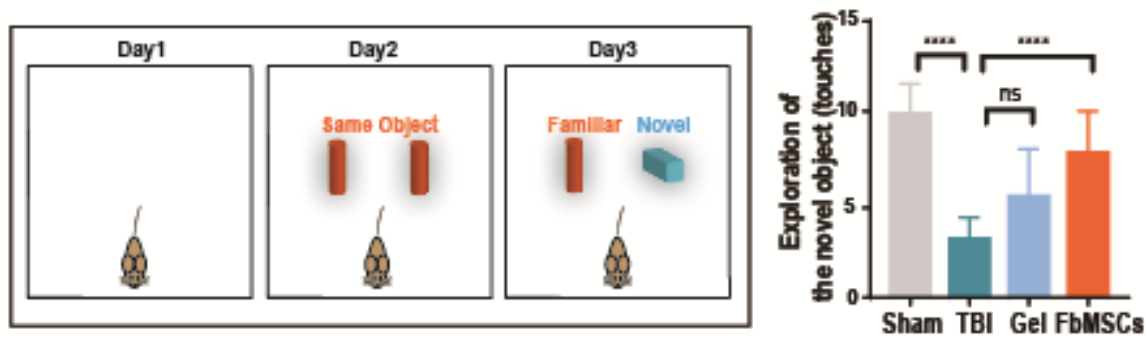

Figure 2

FbMSC transplantation improved learning and cognitive ability in TBI mice. (A) Experimental schedule. FbMSCs were transplanted immediately after TBI (D0). All mice underwent behavioral tests at the indicated days. (B) Schematic of beam walk test (left) and latency to cross the beam (right). (C-F) Morris 
water maze test was used to evaluate the learning and cognitive ability of mice. Typical escape route map (C), learning curve (D), escape latency (E), and movement speed (F) of each group were displayed. Average speed (G) and total distance (H) of each group in the open field test. (I) Schematic (left) and results (right) of new object recognition experiment. Data are presented as the mean \pm standard error; *, $* *, * * *$, and $* * * *$ indicate significance at $p<0.05, p<0.01, p<0.001$, and $p<0.0001$, respectively.

A.

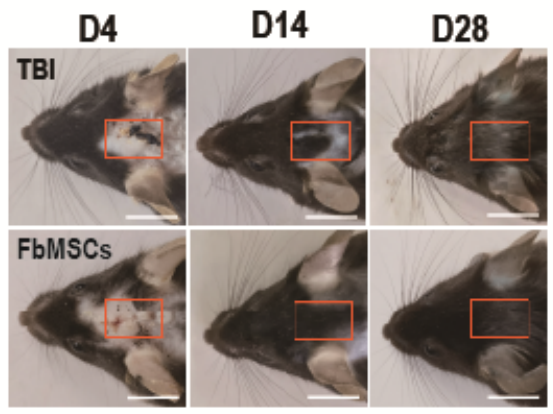

C.

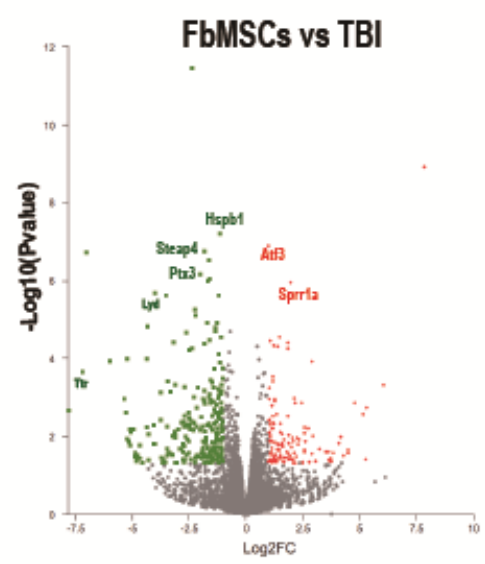

E.

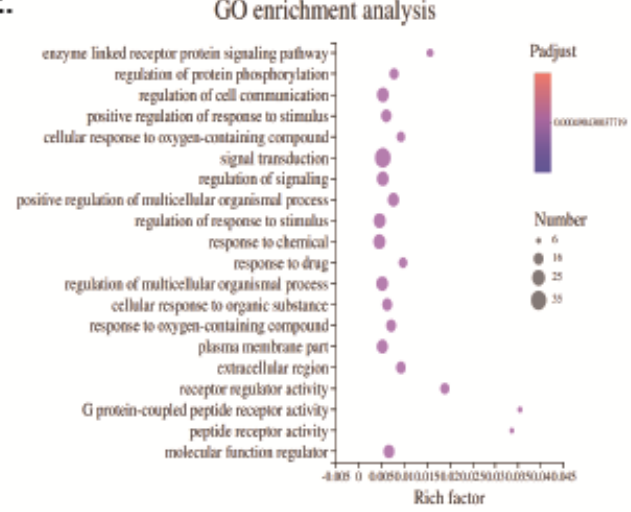

G. Neuroactive ligand-receptor interaction

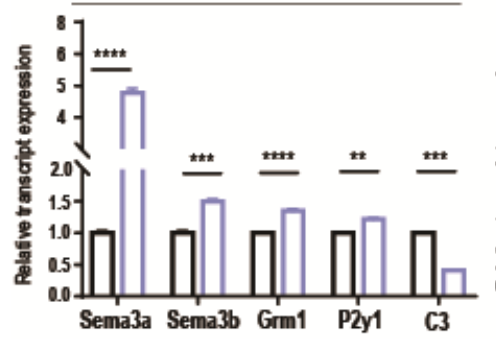

H. IL-17 signaling pathway

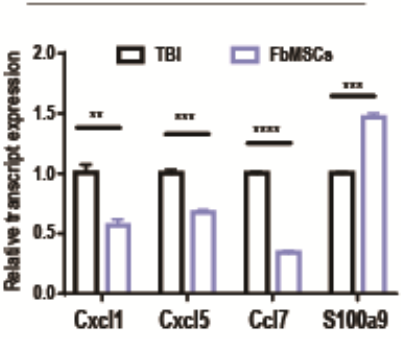

\section{D14 D28}

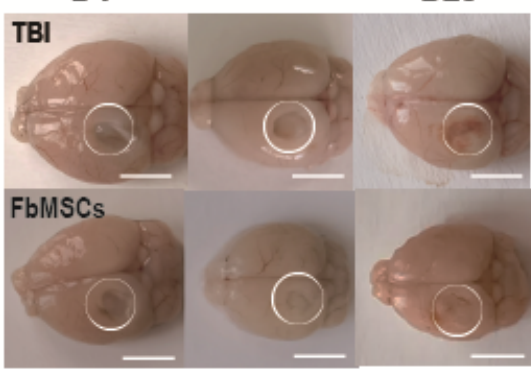

D.

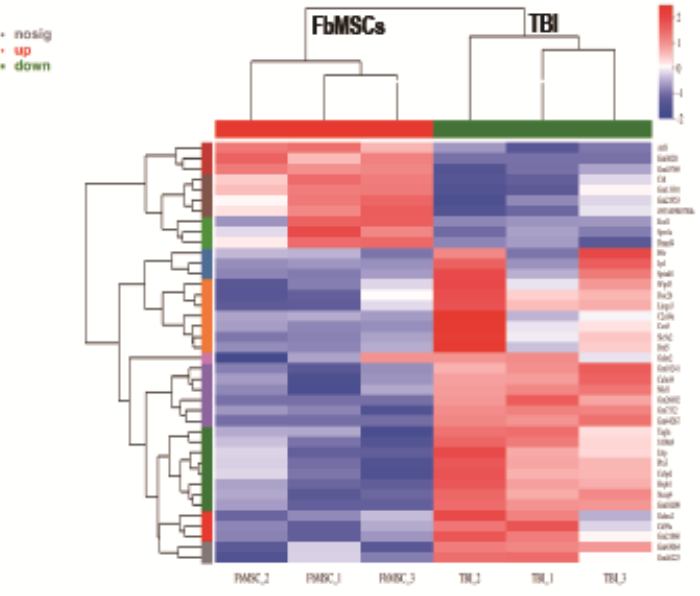

F. KEGG enrichment analysis

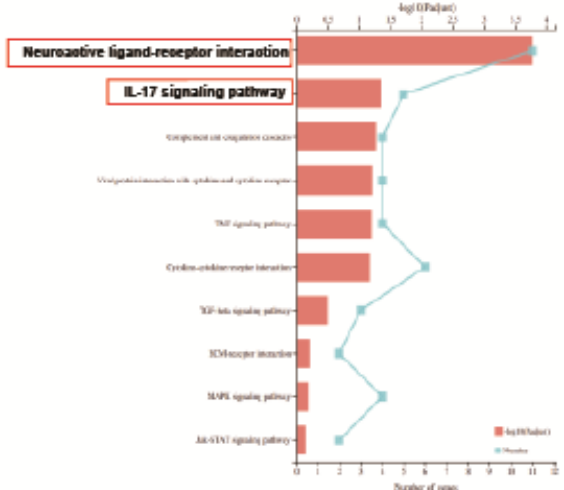

I. MAPK signaling pathway

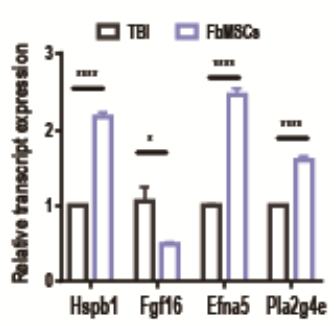

Figure 3 
RNA sequencing analysis of FbMSC-treated TBI group and TBI group. Representative photos of wound healing (A) and topical views of brains (B) from the indicated groups collected at days 4, 14, and 28 after TBI. Scale bar, $2 \mathrm{~mm}$. Volcano maps (C) and (D) heat map of differentially expressed genes in FbMSCtreated TBI group and TBI group. (E) GO analysis showed enrichment in cellular component, molecular function and biological process. (F) KEGG analysis of enriched pathway in FbMSCs. Quantitative RT-PCR verified the expression genes related to neuron ligand-receptor interactions (G), IL-17 signaling pathway $(H)$, MAPK pathway $(\mathrm{I})$. Data are presented as the mean \pm standard error; *, $* \star, * \star \star$, and $* \star \star \star$ indicate significance at $p<0.05, p<0.01, p<0.001$, and $p<0.0001$, respectively. 

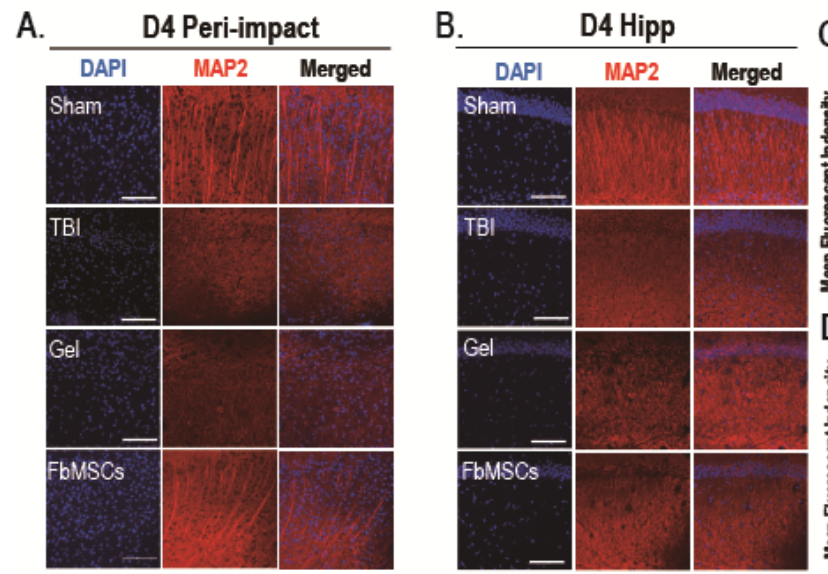

C.

E.

F.
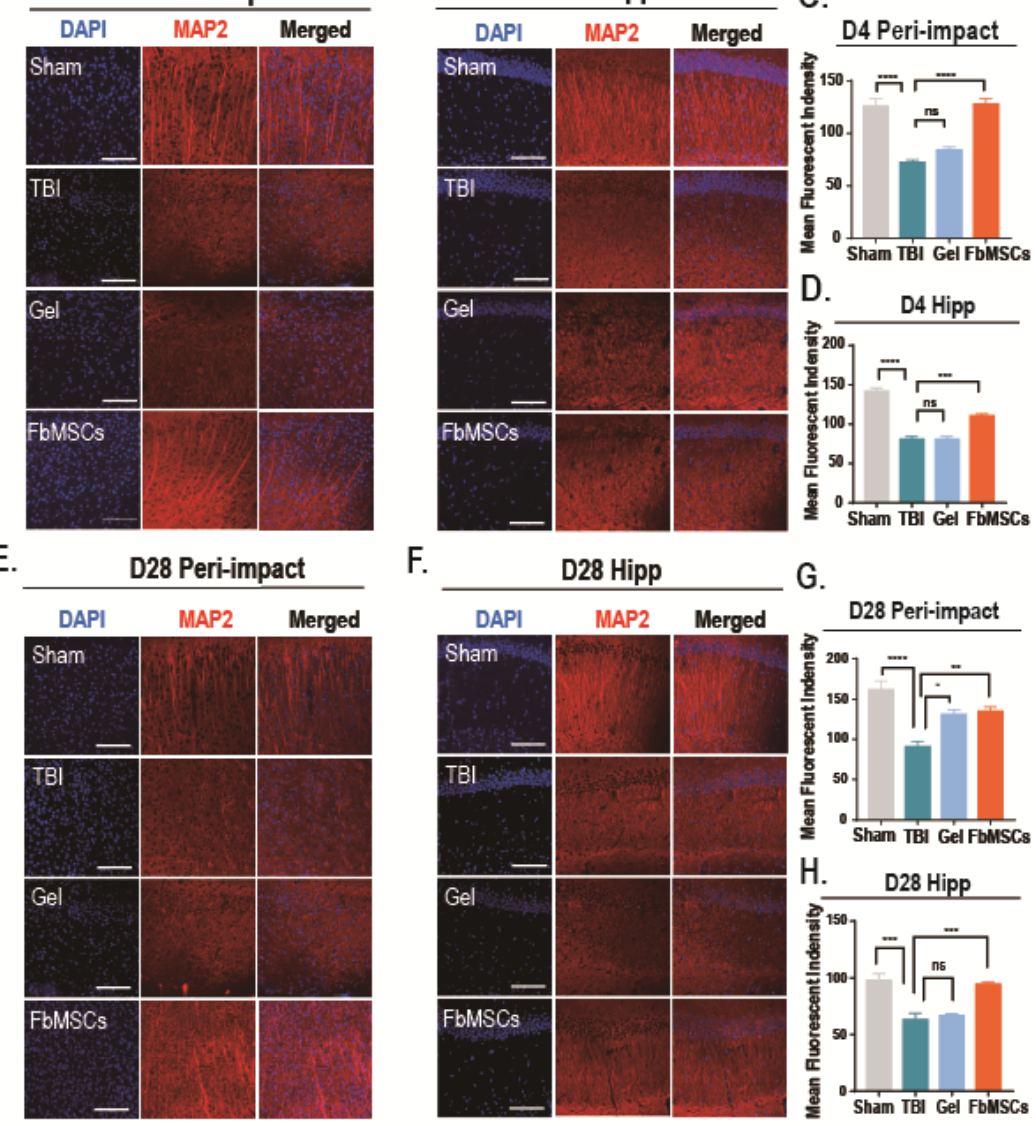

G.

I.

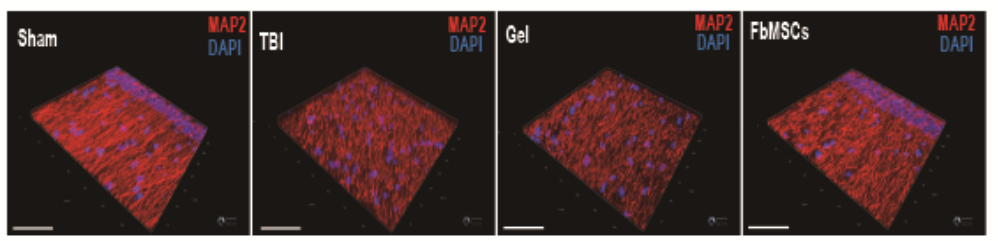

J.
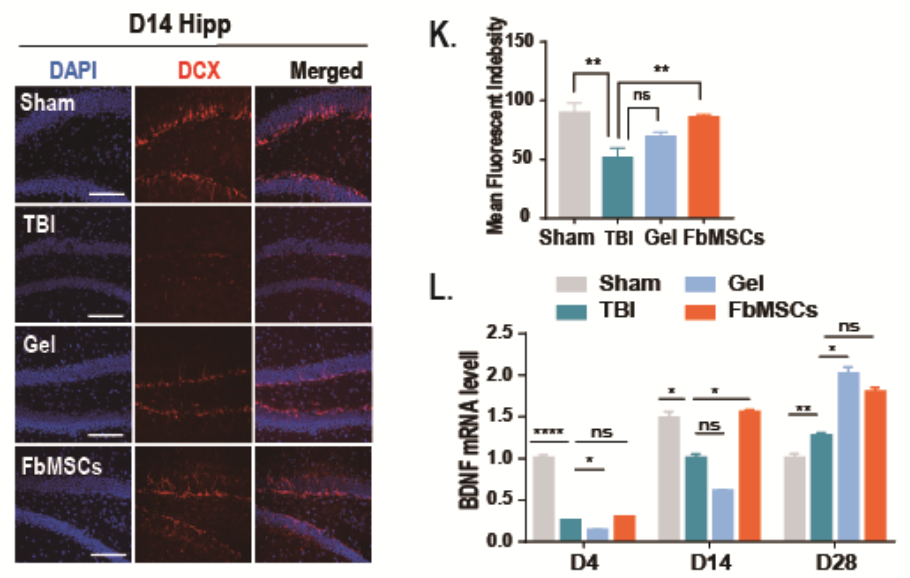

\section{Figure 4}

FbMSCs application promotes the recovery of axonal injury in TBI mice. MAP2 immunofluorescence and fluorescence intensity quantification in peri-impact area and hippocampus in each group at $4 d(A-D)$ and $28 \mathrm{~d}(\mathrm{E}-\mathrm{H})$ post-injury. Scale bar, $20 \mu \mathrm{m}$. (I) Representative 3D reconstruction images of MAP2 staining in hippocampus in each group at $4 \mathrm{~d}$ post-injury. Scale bar, $150 \mu \mathrm{m}$. Doublecortin (DCX) immunofluorescence image $(\mathrm{J})$ and fluorescence intensity quantification $(\mathrm{K})$ in each group at 14d post- 
injury. Scale bar, $20 \mu \mathrm{m}$. (L) Expression of BDNF was detected by qRT-PCR at $4 \mathrm{~d}, 14 \mathrm{~d}$, and $28 \mathrm{~d}$ post-injury. Data are presented as the mean \pm standard error; $*, * \star, * \star \star$, and $* \star \star \star$ indicate significance at $p<0.05, p<$ $0.01, p<0.001$, and $p<0.0001$, respectively.
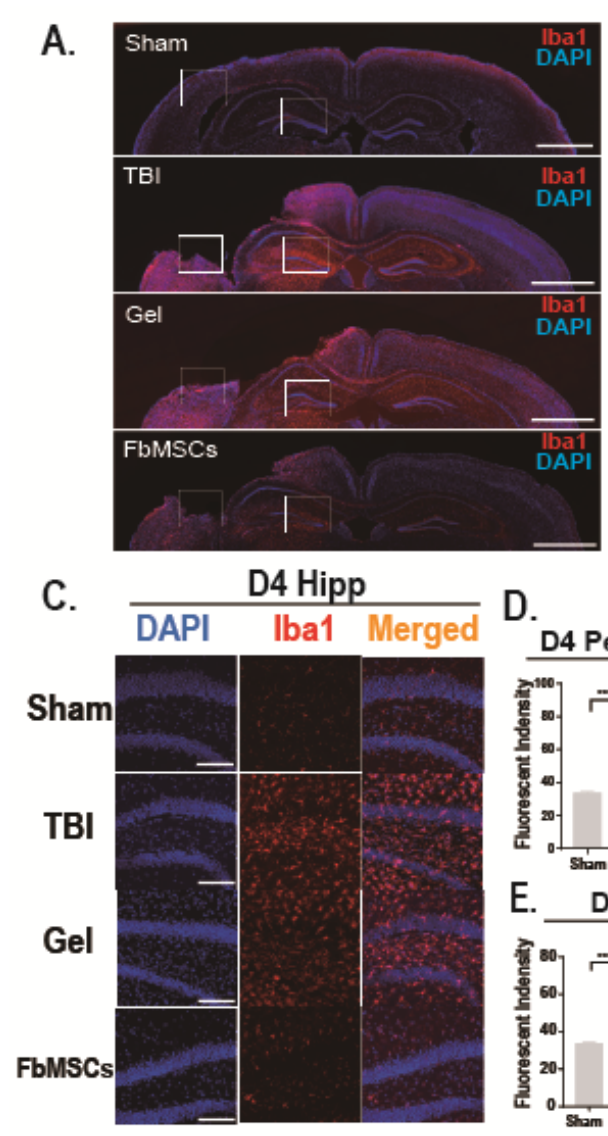

D.
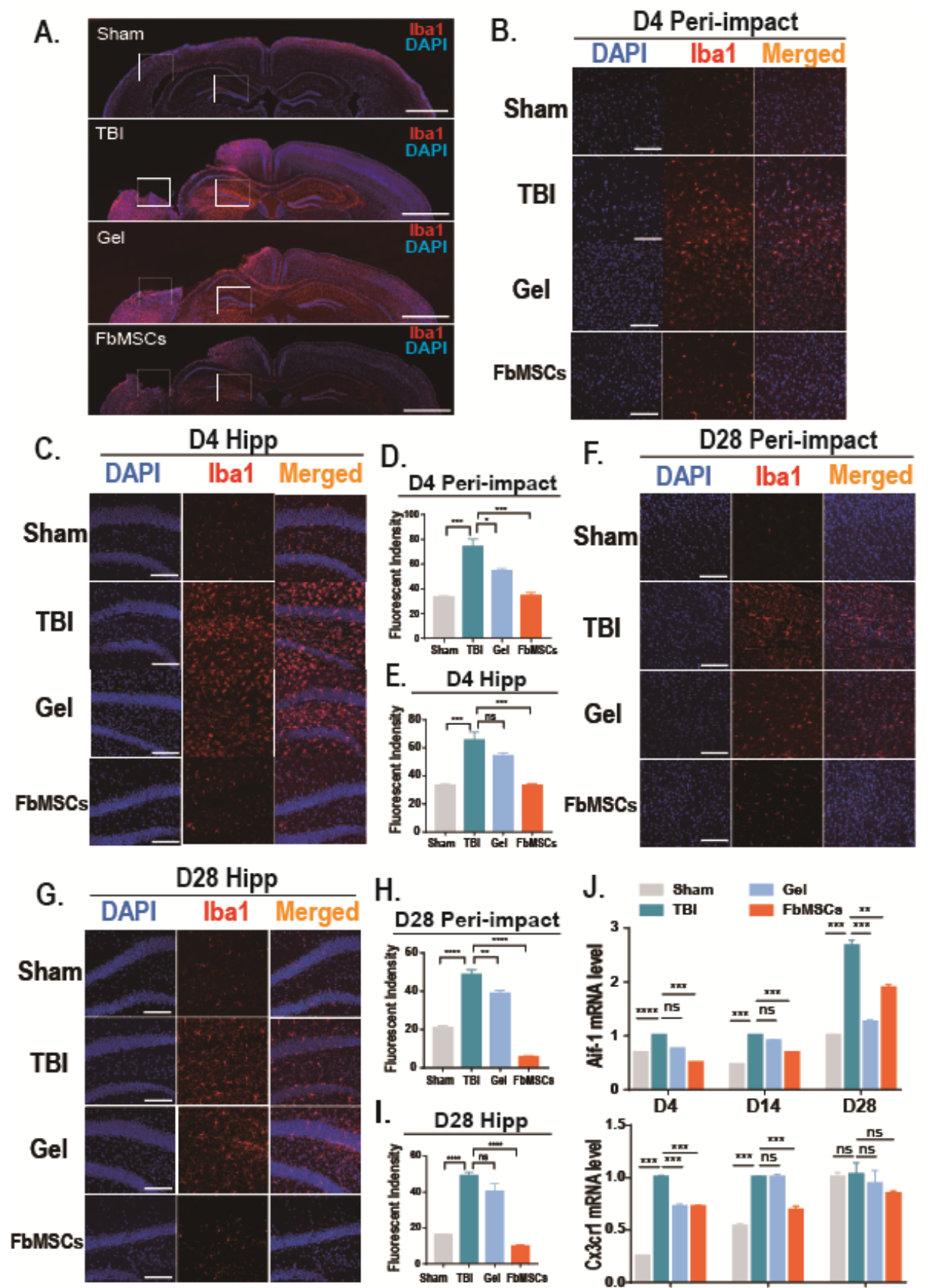

$\mathrm{H}$.
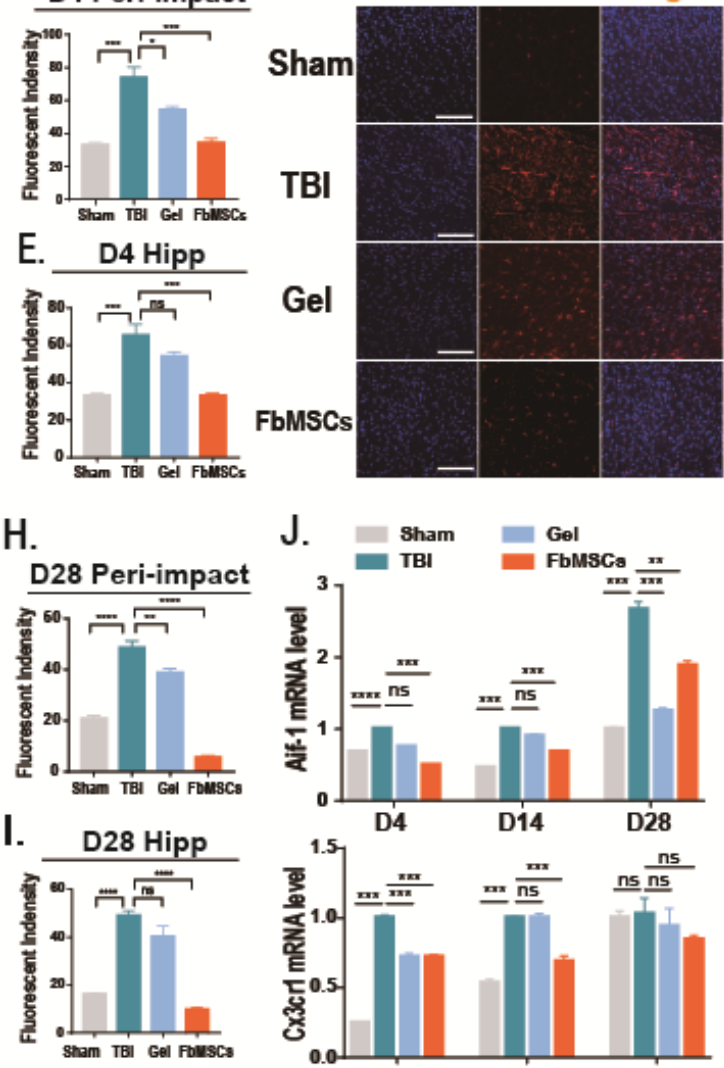

K.
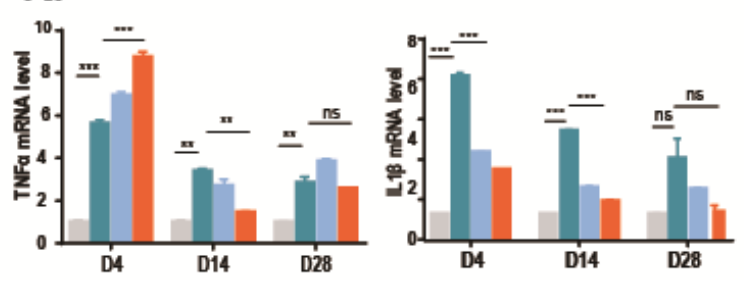

\section{Figure 5}

FbMSCs application decreased the activation of microglia in TBI mice. (A) Representative lba1-stained coronal sections of murine brains of the four groups at $4 \mathrm{~d}$ post-injury. Scale bar, $2 \mathrm{~mm}$. Iba1 
immunofluorescence and fluorescence intensity quantification in peri-impact area and hippocampus in each group at $4 \mathrm{~d}(\mathrm{~B}-\mathrm{E})$ and $28 \mathrm{~d}(\mathrm{~F}-\mathrm{I})$ post-injury. Scale bar, $20 \mu \mathrm{m}$. Expression of AIF1, CX3CR1 $(\mathrm{J})$ and pro-inflammatory factors TNFa, IL1 $1 \beta, \mathrm{IL}-6(\mathrm{~K})$ was detected by qRT-PCR at $4 \mathrm{~d}, 14 \mathrm{~d}$, and $28 \mathrm{~d}$ post-injury. Data are presented as the mean \pm standard error; $*, * \star * \star *$, and $* \star \star \star$ indicate significance at $p<0.05, p<$ $0.01, p<0.001$, and $p<0.0001$, respectively.
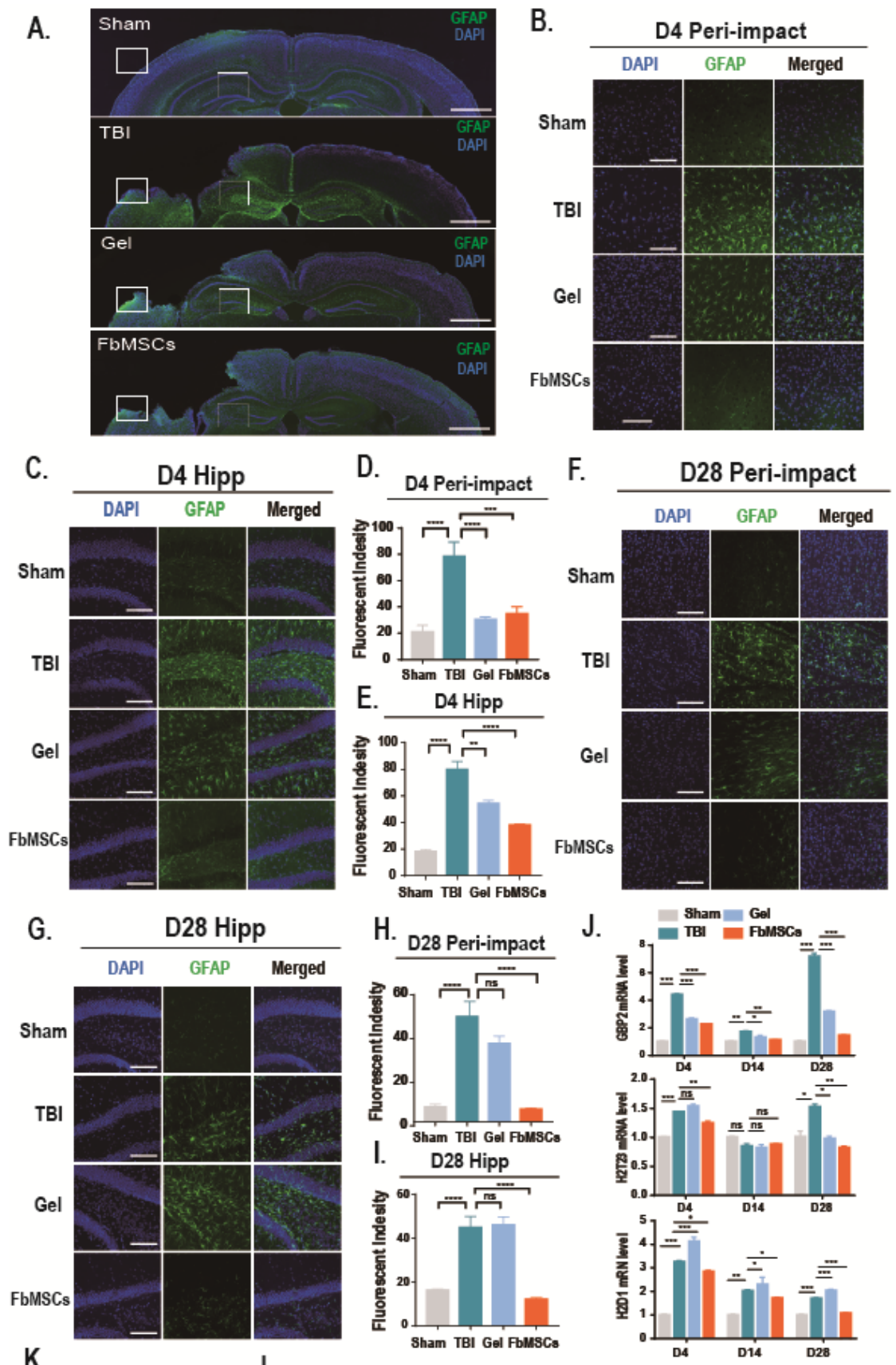

K.

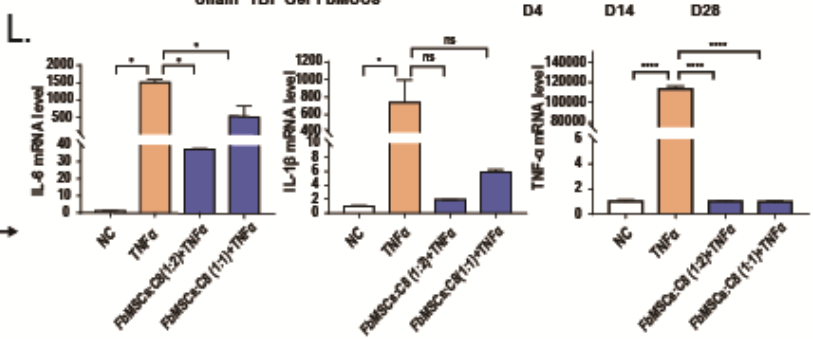

Figure 6 
Application of FbMSCs decreased the activation of astrocytes. (A) Representative GFAP stained coronal sections of murine brains of the four groups at $4 \mathrm{~d}$ post-injury. Scale bar, $2 \mathrm{~mm}$. GFAP immunofluorescence and fluorescence intensity quantification in peri-impact area and hippocampus in each group at $4 \mathrm{~d}(\mathrm{~B}-\mathrm{E}) 28 \mathrm{~d}(\mathrm{~F}-\mathrm{I})$ post-injury. Scale bar, $20 \mu \mathrm{m}$. (J) Expression of H2-T23, H2-D1 and GBP2 was detected by qRT-PCR at $4 \mathrm{~d}, 14 \mathrm{~d}$, and $28 \mathrm{~d}$ post-injury. (K) Schematic of FbMSCs and astrocyte cell line C8-D1A co-culture. (L) C8-D1A cells were collected after $24 \mathrm{~h}$ co-culture, IL-6, IL1 $\beta$, and TNF- $\alpha$

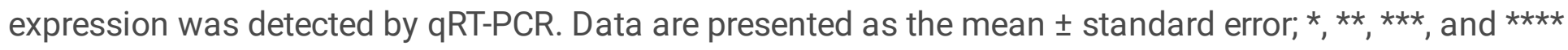
indicate significance at $p<0.05, p<0.01, p<0.001$, and $p<0.0001$, respectively. 
A.

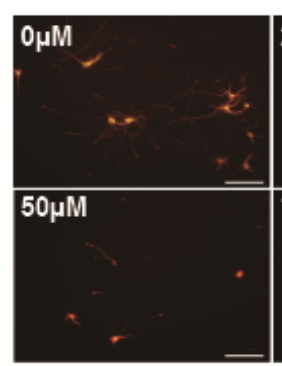

D.

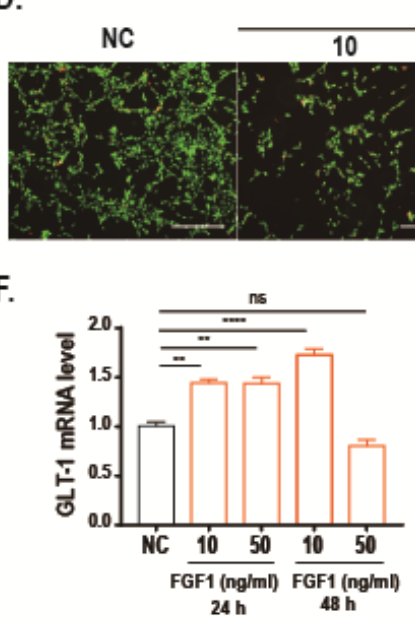

H.

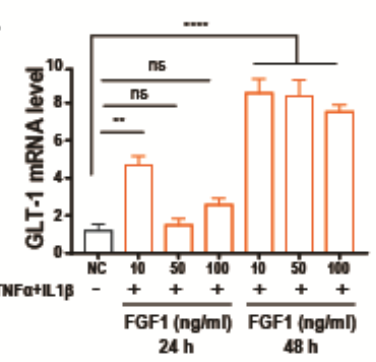

C.
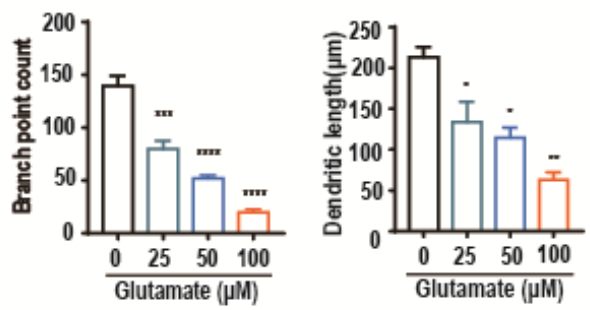

E.

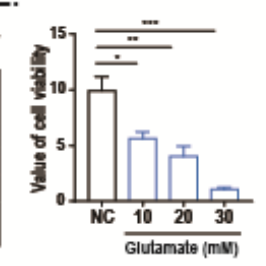

J.
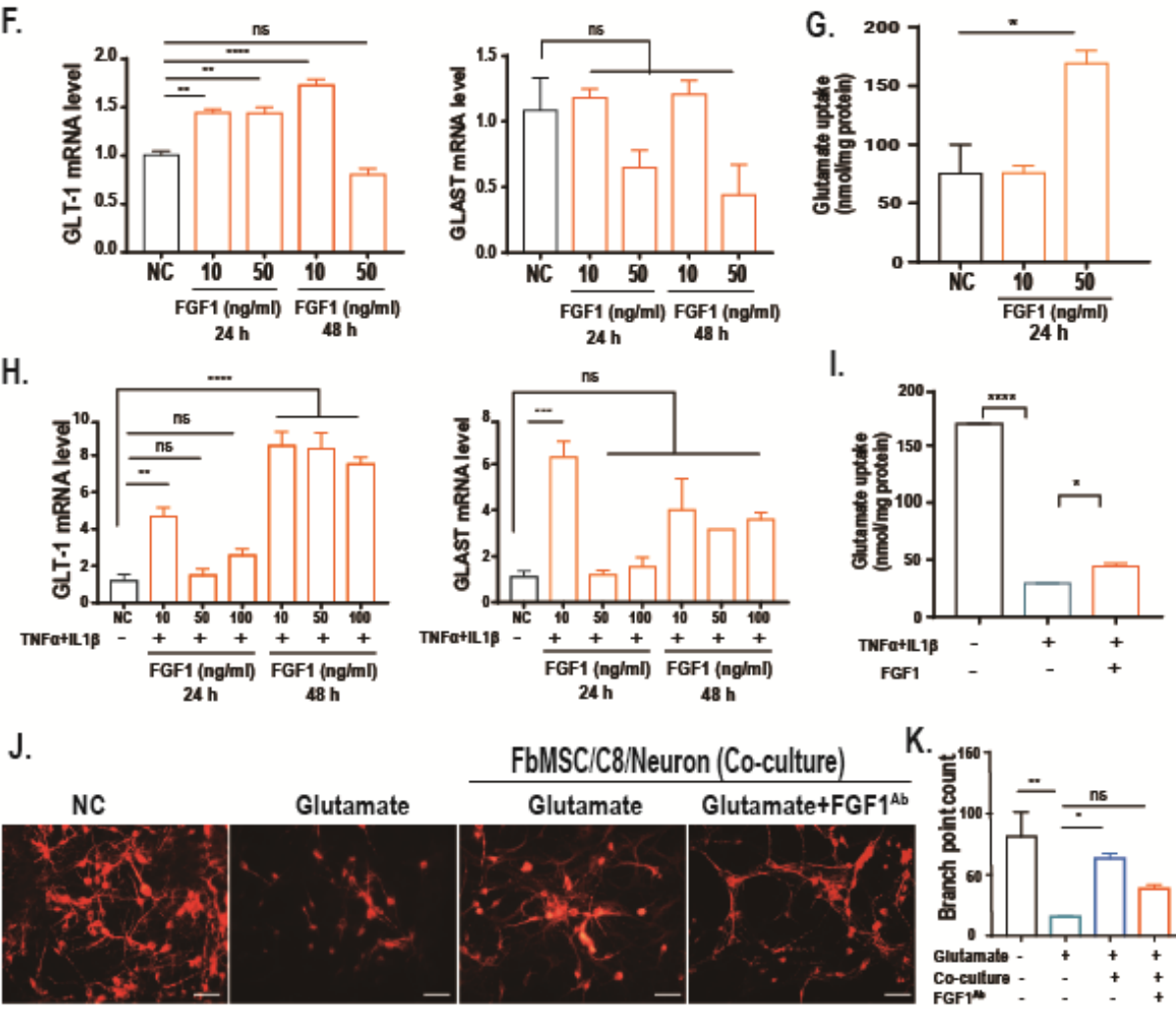

L.

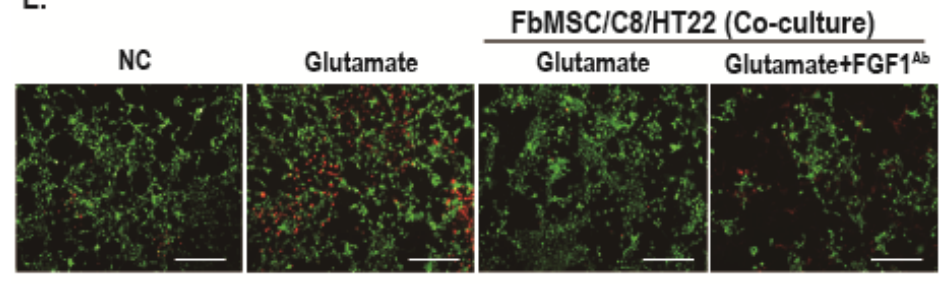

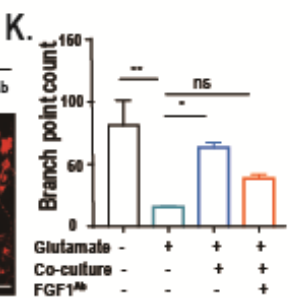

M.

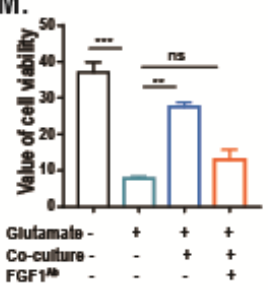

\section{Figure 7}

FGF1 from FbMSCs promotes glutamate uptake of C8-D1A cells and alleviates neuron excitotoxicity. (A) Representative images of MAP2 stained neurons treated without or with indicated doses of glutamate $(100 \mu \mathrm{m})$ for $48 \mathrm{~h}$. Scale bar, $20 \mu \mathrm{m}$. Branch point (B) and Dendritic length (C) were quantified according to MAP2 staining. HT22 cells were treated without or with indicated doses of glutamate $(20 \mathrm{mM})$ for $24 \mathrm{~h}$ and then were stained with calcein-AM (green)/ethidium homodimer (red). Representative staining 
images (D) and quantification of cell viability (E) according to calcein-AM (green)/ethidium homodimer staining. Scale bar, $100 \mu \mathrm{m}$. (F) C8-D1A cells were stimulated without or with indicated doses of FGF1 for $24 \mathrm{~h}$ or $48 \mathrm{~h}$. GLT-1 and GLAST expression was detected by qRT-PCR. (G) Glutamate uptake in C8-D1A cells stimulated without or with indicated doses of FGF1. (H) C8-D1A cells were stimulated without or with TNFa, IL1 $\beta$, and indicated doses of FGF1 for $24 \mathrm{~h}$ or $48 \mathrm{~h}$. GLT-1 and GLAST expression was detected by qRT-PCR. (I) Glutamate uptake in C8-D1A cells stimulated without or with TNFa, IL1 13 , and FGF1. (J) Representative images of MAP2-stained neurons in indicated groups. Scale bar, $20 \mu \mathrm{m}$. (K) Quantification of neuron branches in each group. (L) Representative images of calcein-AM (green)/ethidium homodimer (red) staining of HT22 cells in the indicated groups. Scale bar, $100 \mu \mathrm{m}$. (M) Quantification of cell viability according to calcein-AM (green)/ethidium homodimer staining. Data are presented as the mean \pm

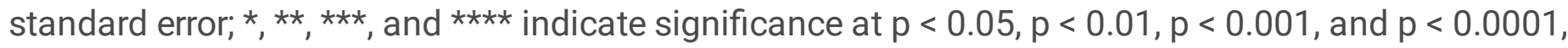
respectively.

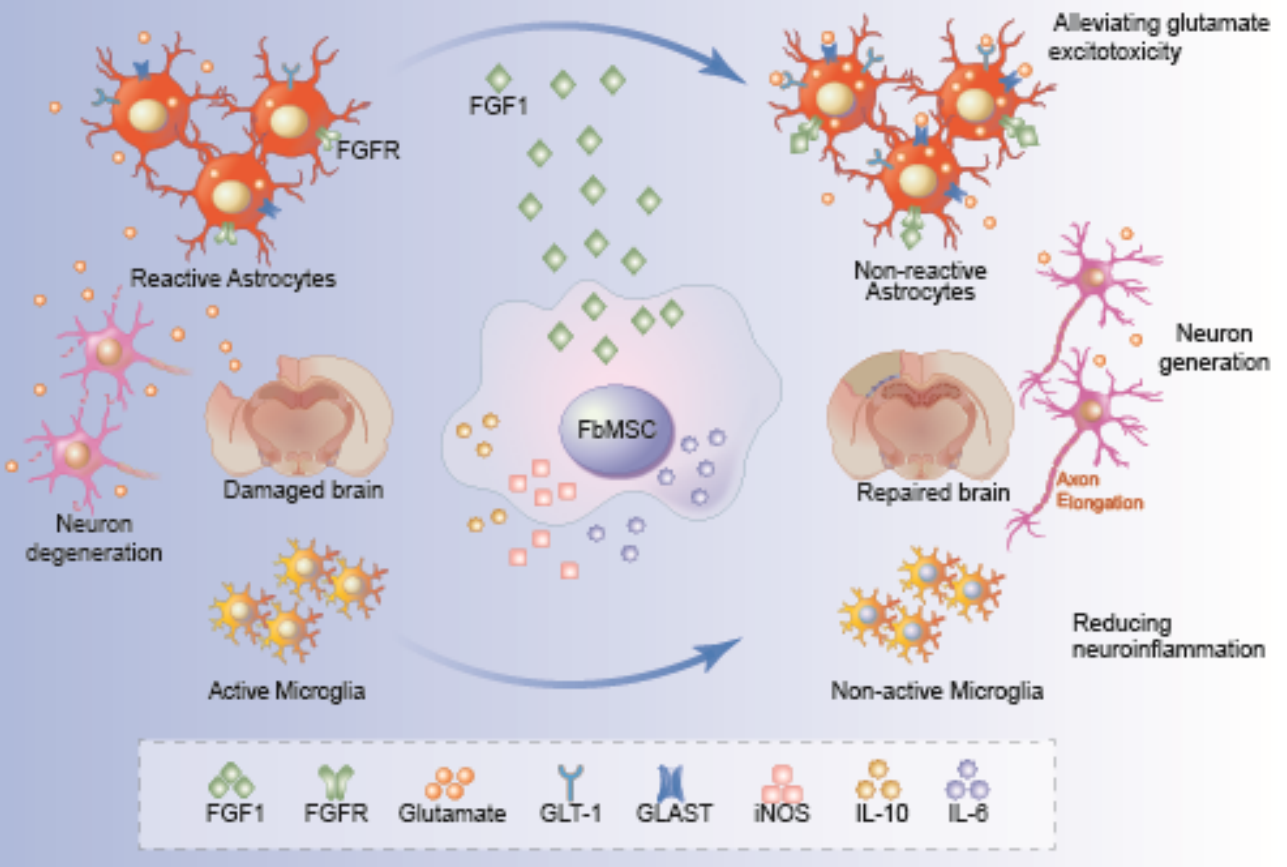

\section{Figure 8}

Proposed mechanism. FbMSC application reduces the activation of microglia cells and astrocytes and alleviates glutamate excitotoxicity. Abbreviations: FGF1, acidic fibroblast growth factor1; FGFR, acidic fibroblast growth factor1 receptor; GLT-1, Glutamate transporter-1; GLAST, glutamate-aspartate transporter; iNOS, inducible nitric oxide synthase; IL-6, interleukin-6; IL-10, interleukin-10.

\section{Supplementary Files}

This is a list of supplementary files associated with this preprint. Click to download. 
- supplementlymaterials.docx 